\title{
Atm deletion with dual recombinase technology preferentially radiosensitizes tumor endothelium
}

\author{
Everett J. Moding, ${ }^{1}$ Chang-Lung Lee, ${ }^{2}$ Katherine D. Castle, ${ }^{1}$ Patrick Oh, ${ }^{2}$ Lan Mao, ${ }^{3}$ Shan Zha, ${ }^{4}$ Hooney D. Min, ${ }^{2}$ Yan Ma, \\ Shiva Das, ${ }^{2}$ and David C. Kirsch ${ }^{1,2}$ \\ 'Department of Pharmacology and Cancer Biology, ${ }^{2}$ Department of Radiation Oncology, and ${ }^{3}$ Department of Medicine, Duke University Medical Center, Durham, North Carolina, USA \\ ${ }^{4}$ Departments of Pathology and Pediatrics, Columbia University, New York, New York, USA.
}

\begin{abstract}
Cells isolated from patients with ataxia telangiectasia are exquisitely sensitive to ionizing radiation. Kinase inhibitors of ATM, the gene mutated in ataxia telangiectasia, can sensitize tumor cells to radiation therapy, but concern that inhibiting ATM in normal tissues will also increase normal tissue toxicity from radiation has limited their clinical application. Endothelial cell damage can contribute to the development of long-term side effects after radiation therapy, but the role of endothelial cell death in tumor response to radiation therapy remains controversial. Here, we developed dual recombinase technology using both FlpO and Cre recombinases to generate primary sarcomas in mice with endothelial cell-specific deletion of Atm to determine whether loss of Atm in endothelial cells sensitizes tumors and normal tissues to radiation. Although deletion of Atm in proliferating tumor endothelial cells enhanced the response of sarcomas to radiation, Atm deletion in quiescent endothelial cells of the heart did not sensitize mice to radiation-induced myocardial necrosis. Blocking cell cycle progression reversed the effect of Atm loss on tumor endothelial cell radiosensitivity. These results indicate that endothelial cells must progress through the cell cycle in order to be radiosensitized by Atm deletion.
\end{abstract}

\section{Introduction}

Approximately $50 \%$ of all cancer patients receive radiation therapy at some point during the course of their illness (1). Despite recent advances in treatment planning and delivery, normal tissue toxicity often limits the ability of radiation to control tumors locally. However, targeted drugs have the potential to increase the probability of tumor eradication by selectively sensitizing tumor cells to radiation $(2,3)$. Ataxia telangiectasia mutated (ATM) is a serine/threonine protein kinase that senses DNA double-strand breaks and phosphorylates several key proteins to initiate the DNA damage response, leading to cell cycle arrest, DNA repair, or apoptosis $(4,5)$. Cells isolated from patients with ataxia telangiectasia, which lack functional ATM, are exquisitely sensitive to ionizing radiation (6). Based on this observation, ATM inhibitors have been developed to sensitize tumors to radiation therapy $(7,8)$. Although these inhibitors have been shown to radiosensitize tumor cell lines and xenografts (9), concern over radiosensitization of normal tissues may limit their clinical application. For example, we previously demonstrated that cell type-specific deletion of the ATM phosphorylation target p53 sensitizes mice to radiation-induced gastrointestinal injury (10) and myocardial necrosis (11). Although

\section{Related Commentary: p. 3289}

Note regarding evaluation of this manuscript: Manuscripts authored by scientists associated with Duke University, The University of North Carolina at Chapel Hill, Duke-NUS, and the Sanford-Burnham Medical Research Institute are handled not by members of the editorial board but rather by the science editors, who consult with selected external editors and reviewers.

Conflict of interest: The authors have declared that no conflict of interest exists. Submitted: April 2, 2014; Accepted: May 15, 2014.

Reference information: J Clin Invest. 2014;124(8):3325-3338. doi:10.1172/JCI73932.
Atm-knockout mice have been used to study acute radiation injury, these mice are tumor prone $(12,13)$. Therefore, this model cannot be used to investigate the long-term consequences of blocking ATM during focal radiation therapy.

Human cancers develop in a complex environment composed of blood vessels, fibroblasts, and immune cells. The tumor microenvironment contributes to cancer development, progression, and response to therapy (14). However, it remains controversial whether stromal cells, such as endothelial cells, can be targeted by radiosensitizers to enhance the efficacy of radiation therapy (15-17). It has been reported that deletion of Atm in endothelial cells impairs the development of tumor vasculature and slows the growth of transplanted melanoma cell lines (18). However, transplanted tumor models may not fully recapitulate the vasculature of autochthonous tumors (19). To clarify the function of endothelial cell ATM in tumor development and radiation response, and also to investigate the long-term consequences of blocking ATM in normal tissues treated with focal radiation therapy, we have developed novel dual recombinase technology to mutate Atm specifically in the endothelial cells of primary tumors (20). Our findings demonstrated that loss of Atm in endothelial cells did not affect tumor initiation or growth of primary soft tissue sarcomas. However, deletion of Atm specifically in proliferating tumor endothelial cells increased radiation-induced endothelial cell death and delayed tumor regrowth after radiation therapy. In contrast, deletion of Atm in quiescent cardiac endothelial cells did not sensitize mice to radiation-induced myocardial necrosis. Although loss of Atm radiosensitized p53-null endothelial cells in vitro and tumor endothelial cells in vivo, radiosensitization was reversed by treatment with a cyclin-dependent kinase inhibitor. These results suggest that the effect of Atm loss on radiosensitivity depends on the state of cellular proliferation. 
A Intramuscular Adeno-FlpO; VE-Cadherin-Cre
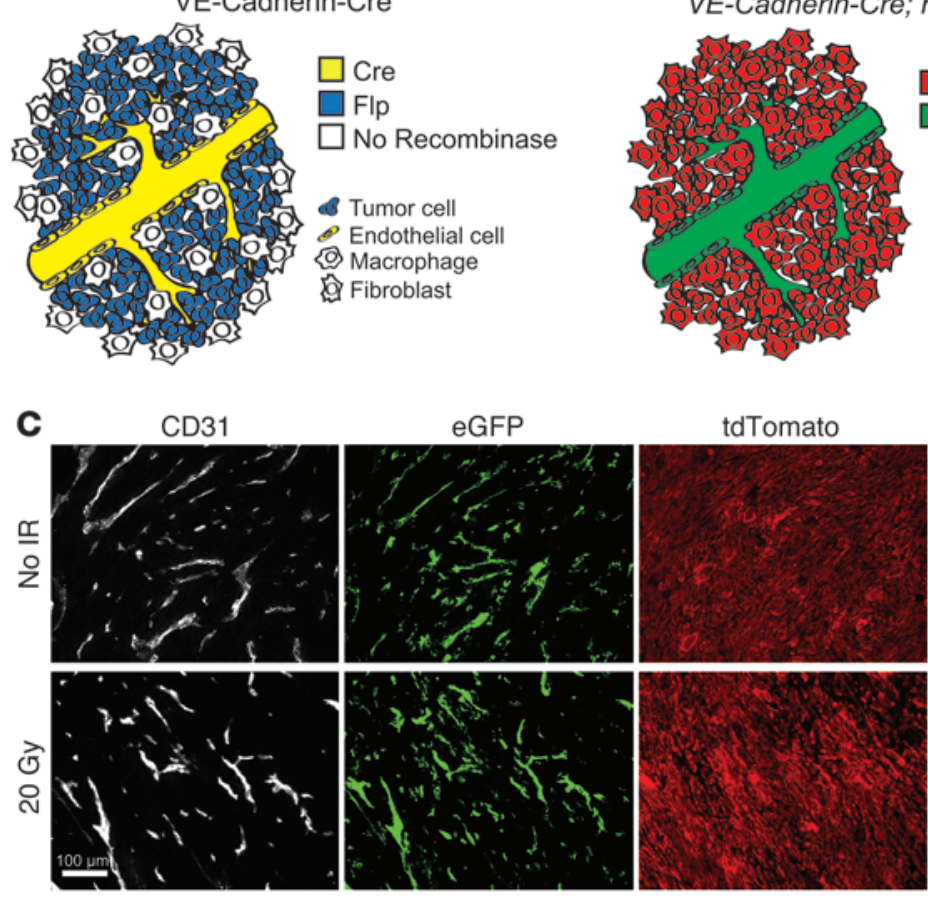

E

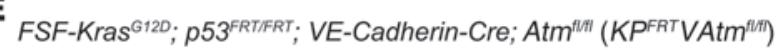
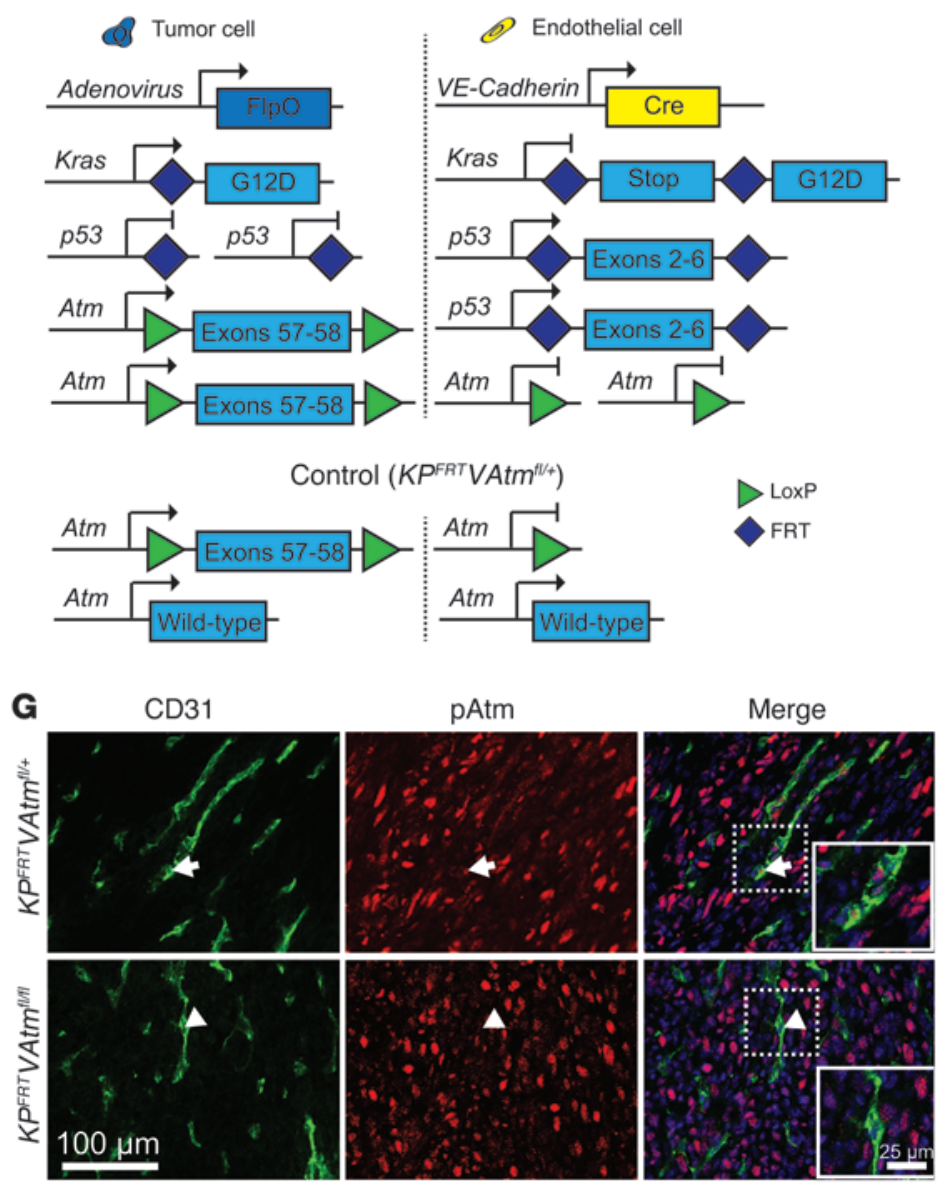
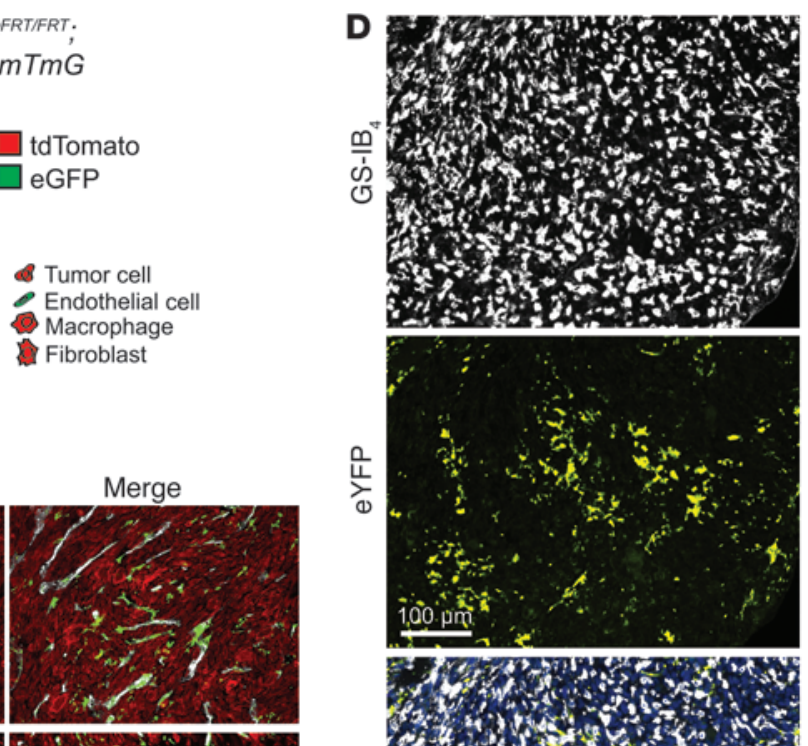

Tumor cell

Macrophage

Aibroblast

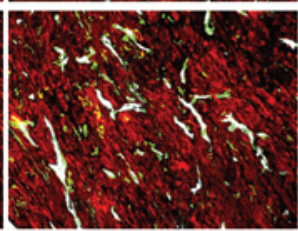

F

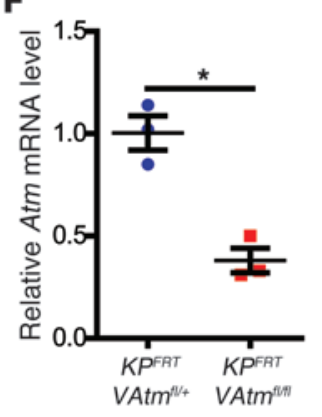

H

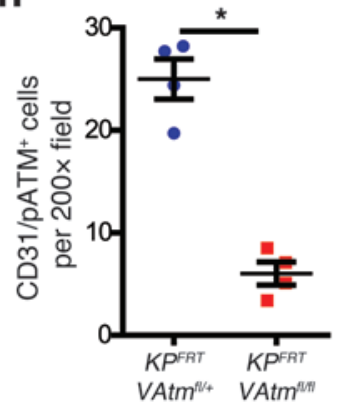


Figure 1. Dual recombinase technology enables VE-Cadherin-Cre to delete Atm in primary sarcoma endothelial cells. (A) Recombinase expression in $K P^{F R T}$; VE-Cadherin-Cre mice injected with adeno-FIpO to generate sarcomas. (B) Reporter expression in KP ${ }^{F R}$; VE-Cadherin-Cre; $\mathrm{mTmG}$ mice. All cells initially express tdTomato, and VE-Cadherin-Cre deletes tdTomato and turns on eGFP expression in endothelial cells (green). (C) Fluorescence images of CD31-stained soft tissue sarcomas initiated with adeno-FlpO in KPFRT; VE-Cadherin-Cre; $m T M G$ mice in the absence of radiation (No IR) and 2 weeks after irradiation with $20 \mathrm{~Gy}$. Images are representative of 3 mice per group. (D) Representative immunofluorescence images of a sarcoma in a KPloxp; LSL-eYFP mouse initiated with adeno-Cre and stained with GS-IB ${ }_{4}$. (E) Genetic strategy to activate Kras and delete $p 53$ in tumor cells and delete Atm in endothelial cells. Control mice retained 1 WT allele of Atm in endothelial cells. (F) Expression of Atm mRNA in FACS-isolated tumor endothelial cells (CD45-CD34+CD31+) from the indicated mice $(n=3$ per group). (G and $\mathbf{H})$ Immunofluorescence (G) and quantification (H) of

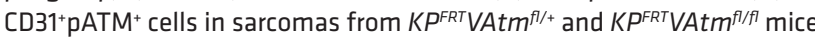
4 hours after irradiation with $20 \mathrm{~Gy}\left(n=4\right.$ per group). A pATM ${ }^{+}$endothelial cell in the KPFRTVAtm ${ }^{f /+}$ mouse (arrows) and a pATM- endothelial cell in the $K P^{F R T} V A t m^{f / / f l}$ mouse (arrowheads) are shown at higher magnification in the insets. Data are mean \pm SEM. Scale bars: $100 \mu \mathrm{m}$ (C, D, and G); $25 \mu \mathrm{m}$ (C, insets) ${ }^{*} P<0.05$.

\section{Results}

Deletion of Atm in endothelial cells of primary tumors. It has recently been reported that ATM is activated in the endothelial cells of transplanted melanomas, and that deletion of Atm in endothelial cells decreases tumor angiogenesis and slows tumor growth (18). To investigate ATM activity in the endothelial cells of primary soft tissue sarcomas, we generated sarcomas in conditional LSL-Kras ${ }^{G 12 D}$; $p 53^{f / f}$ mice (referred to herein as $K P^{l o x P}$; ref. 21 ) and collected unirradiated tumors and tumors 4 hours after irradiation of the sarcoma with 20 Gy (Supplemental Figure 1A; supplemental material available online with this article; doi:10.1172/JCI73932DS1). We selected $20 \mathrm{~Gy}$ for our studies because this dose of radiation has been previously reported to trigger radiation-induced endothelial cell death in normal tissues (22) and tumors (15). 20 Gy is also a radiation dose used in the clinic to treat cancers with radiosurgery or stereotactic body radiation therapy. We stained sections from these tumors with antibodies against the endothelial cell surface marker CD31, ATM phosphorylated at Ser1987 (pATM), and KAP1 phosphorylated at Ser824 (pKAP1), as KAP1 is phosphorylated by ATM in response to DNA damage (23). Consistent with the previous report in transplanted melanomas (18), we detected ATM activation in unirradiated sarcoma endothelial cells. In addition, pATM and pKAP1 levels in tumor endothelial cells were significantly increased after $20 \mathrm{~Gy}$ focal irradiation (Supplemental Figure 1, B-E).

To investigate the role of ATM in the endothelial cells of primary tumors, we used dual recombinase technology to initiate sarcomas in conditional FSF-Kras ${ }^{G 12 D}$; $p 53^{F R T / F R T}$ (referred to herein as $K P^{F R T}$ ) mice with an adenovirus expressing FlpO (adeno-FlpO) (20) and deleted Atm floxed alleles $\left(\mathrm{Atm}^{f}\right)$ (24) in endothelial cells with VECadherin-Cre (Figure 1A and ref. 25). We evaluated the efficiency of Cre-mediated recombination in tumor endothelial cells by crossing $K P^{F R T}$; VE-Cadherin-Cre mice to membrane-Tomato/membraneGFP ( $m T m G)$ reporter mice (26). In $m T m G$ mice, cells expressing Cre are labeled with enhanced GFP (eGFP), and cells that do not express Cre are labeled with tdTomato protein (Figure 1B).
To determine whether eGFP is expressed in tumor endothelial cells from $K P^{F R T}$; VE-Cadherin-Cre; $m T m G$ mice, we stained sarcomas with the endothelial cell markers CD31, VE-Cadherin, and CD34 and the myeloid cell marker CD11b. The majority of eGFP-expressing cells also expressed the endothelial cell markers, and very few myeloid cells expressed eGFP (Figure $1 \mathrm{C}$ and Supplemental Figure 2, A-C).

To confirm that tumors initiated by intramuscular injection of adenovirus only have Kras and p53 mutated in tumor cells, we generated sarcomas with an adenovirus expressing Cre recombinase in $K P^{l o x P}$ mice with a Cre-activated eYFP reporter $(L S L-e Y F P)(27)$. Staining with Griffonia simplicifolia- $\mathrm{IB}_{4}$ lectin $\left(\mathrm{GS}-\mathrm{IB}_{4}\right)$ to label endothelial cells (28) and macrophages (29) confirmed that tumor cells had recombined the eYFP reporter, while the stromal cells were not infected by the adenovirus (Figure 1D).

To evaluate the efficiency of deleting the $\mathrm{Atm}^{f}$ allele in primary sarcoma endothelial cells with VE-Cadherin-Cre, we generated sarcomas in $K P^{F R T}$; VE-Cadherin-Cre; Atm ${ }^{A / A}$ (referred to herein as $\left.K P^{F R T} V A t m^{f / f l}\right)$ mice and in control $K P^{F R T} V A t m^{f /+}$ littermates, which retain $1 \mathrm{WT}$ allele of Atm in endothelial cells (Figure 1E). We isolated endothelial cells from sarcomas in $K P^{F R T} V A t m^{A /+}$ and $K P^{F R T} V A t m^{f / A l}$ mice by fluorescence-activated cell sorting (FACS) (Supplemental Figure 2D) and measured Atm mRNA levels by quantitative real-time RT-PCR (qRT-PCR). Tumor endothelial cells from $K P^{F R T} V A t m^{A / A}$ mice had significantly less Atm mRNA than tumor endothelial cells from $K P^{F R T} V A t m^{A /+}$ controls (Figure $1 \mathrm{~F}$ ). In addition, pATM and pKAP1 were significantly reduced in CD31+ cells after $20 \mathrm{~Gy}$ focal irradiation of primary sarcomas in $K P^{F R T}$ $V A t m^{A / A}$ mice (Figure 1, G and $\mathrm{H}$, and Supplemental Figure 2, E and F), which suggests that VE-Cadherin-Cre functionally deletes Atm in sarcoma endothelial cells.

We next investigated the effect of Atm deletion in tumor endothelial cells on tumor initiation and growth. In contrast to transplanted melanomas (18), there was no change in primary sarcoma initiation or sarcoma growth after deletion of Atm in endothelial cells (Figure 2, A-C, and Supplemental Figure 3, A-C). Moreover, microvascular density and tumor hypoxia in primary sarcomas were not affected by deletion of Atm in tumor endothelial cells (Figure 2, D-G, and Supplemental Figure 3, D and E), demonstrating that Atm in endothelial cells is not essential for angiogenesis in primary soft tissue sarcomas.

Loss of Atm sensitizes tumor endothelial cells to radiation therapy. To determine the effect of Atm deletion in endothelial cells on tumor radiation response, we irradiated sarcomas in $K P^{F R T}$ $V_{A t m^{A /+}}$ and $K P^{F R T} V A t m^{f / f l}$ mice with a single dose of $20 \mathrm{~Gy}$ using fluoroscopy-guided radiation therapy. Deletion of Atm significantly increased the number of dead endothelial cells 24 hours after radiation therapy, as indicated by CD31 and TUNEL double staining, but did not affect endothelial cell apoptosis 4 hours after radiation therapy (Figure 3, A and B, and Supplemental Figure 4A). In addition, loss of Atm in endothelial cells increased the total number of TUNEL ${ }^{+}$cells within the sarcoma at 24 hours after irradiation (Figure 3C), which suggests that endothelial cell death in primary sarcomas may trigger death of adjacent cells. Because the timing of endothelial cell death in sarcomas from $K P^{F R T} V A t m^{f / f l}$ mice was consistent with mitotic catastrophe, and because cells undergoing mitotic catastrophe can trigger the cellular apoptotic 


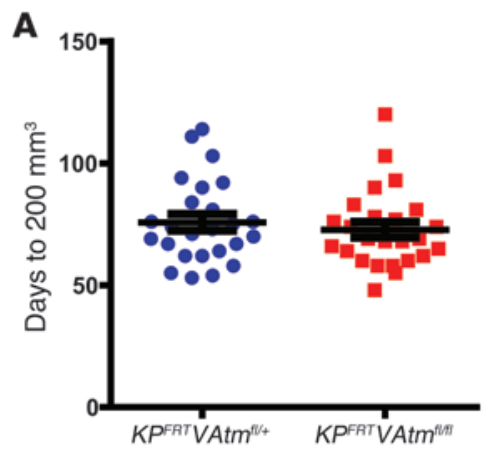

D

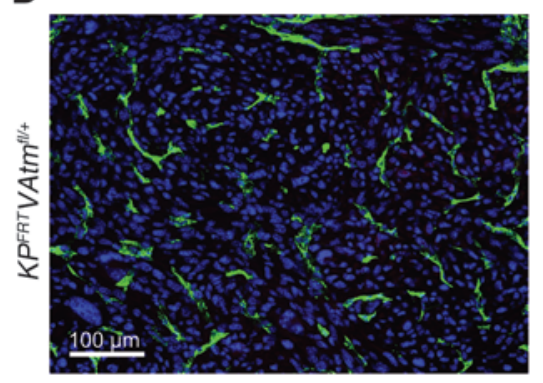

$\mathbf{F}$

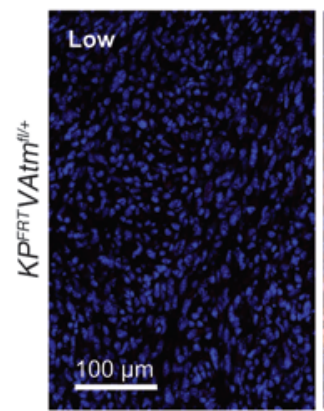

B
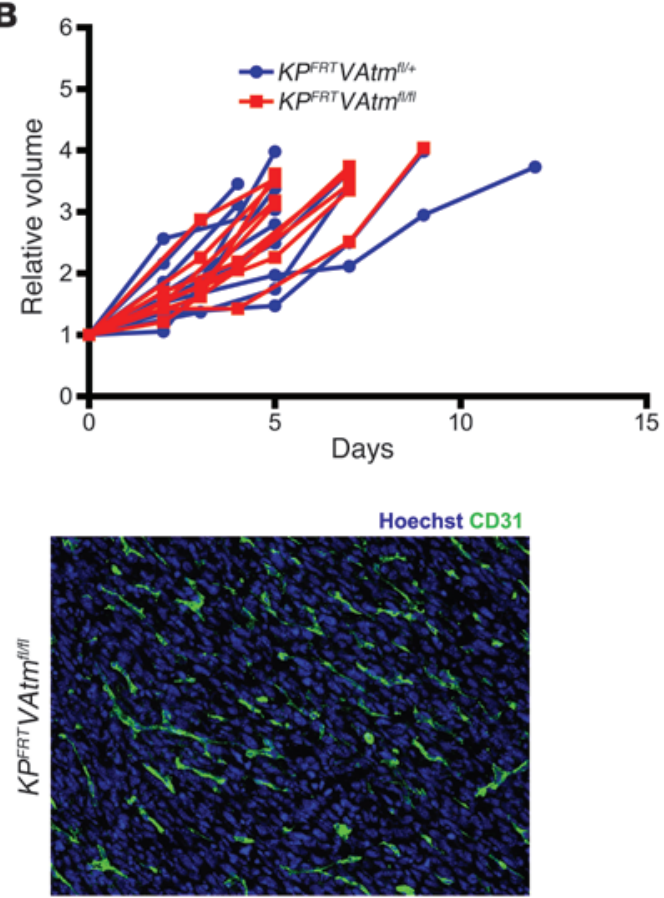

Hoechst EF5
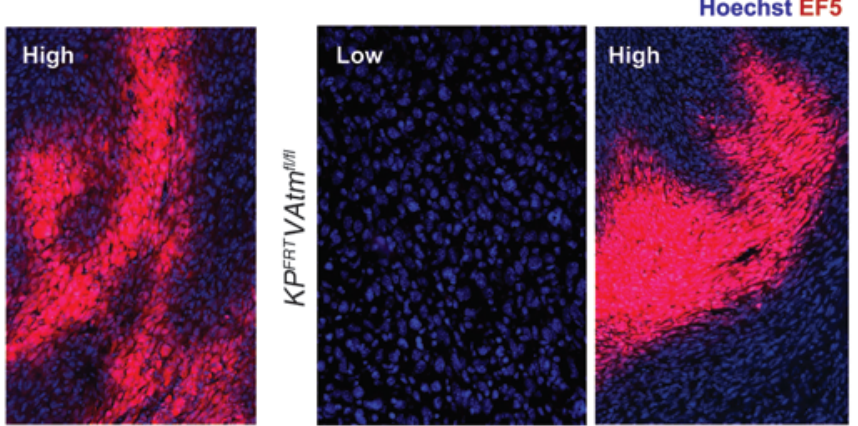
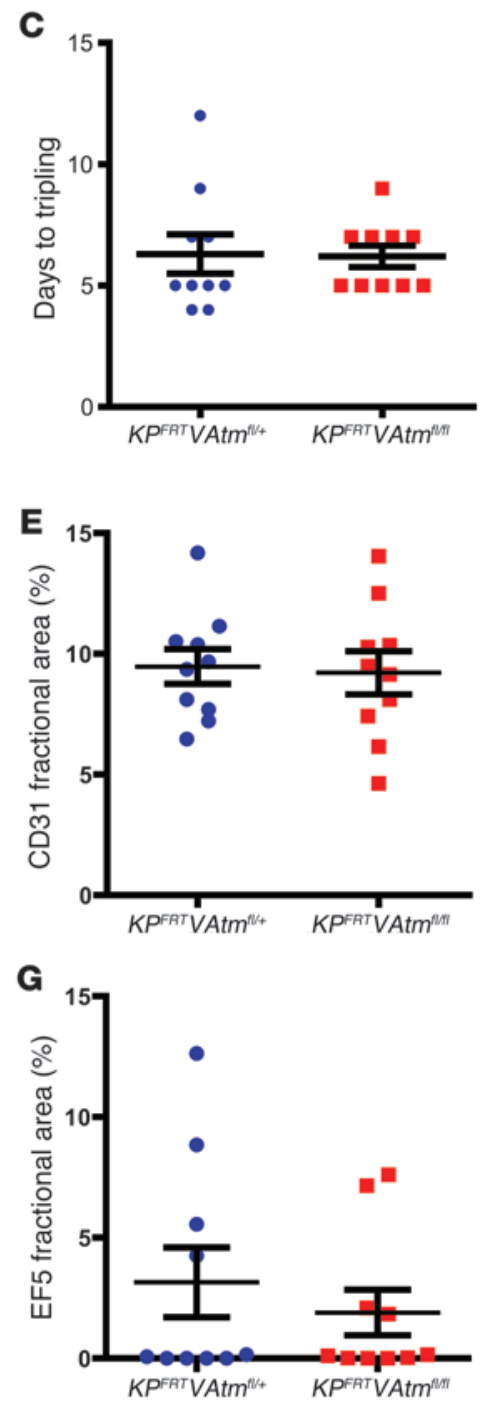

Figure 2. Deletion of Atm in endothelial cells does not affect tumor growth or vascular development of primary soft tissue sarcomas. (A) Time for sarcomas to reach $200 \mathrm{~mm}^{3}$ after intramuscular injection of adeno-FlpO in $K P^{F R T} V A t m^{f /+}$ and $K P^{F R T} V A t m^{f / f l}$ mice $(n=25$ per group). (B) Tumor growth curves and (C) time to tripling for unirradiated sarcomas in $K P^{F R T} V A t m^{f /+}$ and $K P^{F R T} V A t m^{f / / f}$ mice $(n=10$ per group). (D and $\mathbf{E})$ Immunofluorescence (D) and quantification (E) of endothelial cell marker CD31 in sarcomas from $K P^{F R T} V A t m^{f /+}$ and $K P^{F R T} V A t m^{f / / f}$ mice $(n=10$ per group). (F and $\mathbf{G})$ Immunofluorescence $(\mathbf{F})$ and quantification (G) of hypoxia marker EF5 in sarcomas from $K P^{F R T} V A t m^{f /+}$ and $K P^{F R T} V A t m^{f / f l}$ mice ( $n=10$ per group). Regions of low and high hypoxia are shown for both genotypes. All data are mean \pm SEM. Scale bars: $100 \mu \mathrm{m}$ (D and F).

machinery (30), we also stained the sarcomas from $K P^{F R T} V A t m^{f /+}$ and $K P^{F R T} V A t m^{f / f l}$ mice with cleaved caspase 3 (CC3). Deletion of Atm significantly increased the number of $\mathrm{CD}^{+} 1^{+} \mathrm{CC}^{+}$cells 24 hours after radiation therapy (Figure 3D and Supplemental Figure 4B). Taken together, these data show that loss of Atm radiosensitizes tumor endothelial cells in vivo.

To investigate functional changes in tumor vasculature after radiation, we imaged sarcomas in $K P^{F R T} V A t m^{f /+}$ and $K P^{F R T}$ $V A t^{f / f l}$ mice using fluorescence molecular tomography (FMT) with AngioSense, a blood pool imaging agent (31). Prior to irradiation and 24 hours after treatment with $20 \mathrm{~Gy}$, AngioSense was injected intravenously, and the concentration of accumulated probe within each sarcoma was measured after 24 hours. In contrast to tumors in $K P^{F R T} V A t m^{f /+}$ mice, which had an increase in AngioSense accumulation after irradiation, AngioSense accumu- lation decreased in tumors from $K P^{F R T} V A t m^{A / f l}$ mice (Figure 3, E and $F$ ), suggestive of decreased vascular function. To determine whether the increased radiation-induced endothelial cell death in tumors from $K P^{F R T} V A t m^{f / A}$ mice decreased blood flow to tumors, we injected Hoechst 33342 intravascularly and quantified tumor perfusion. Hoechst 33342 perfusion was significantly decreased in sarcomas from $K P^{F R T} V A t m^{f / f l}$ mice 24 hours after irradiation with 20 Gy (Figure $3 \mathrm{G}$ and Supplemental Figure 4C).

We next evaluated whether endothelial cell death contributes to the radiation response of primary sarcomas by irradiating tumors with $20 \mathrm{~Gy}$. After radiation, we monitored tumor growth until the tumors tripled in size. $K P^{F R T} V A t m^{f / f l}$ tumors had a significantly longer growth delay than $K P^{F R T} V A t m^{f /+}$ tumors (Figure 3, H and I), taking $55 \%$ longer to triple in size. We also repeated this experiment by treating primary sarcomas with 10 daily fractions of $3 \mathrm{~Gy}$, model- 
A
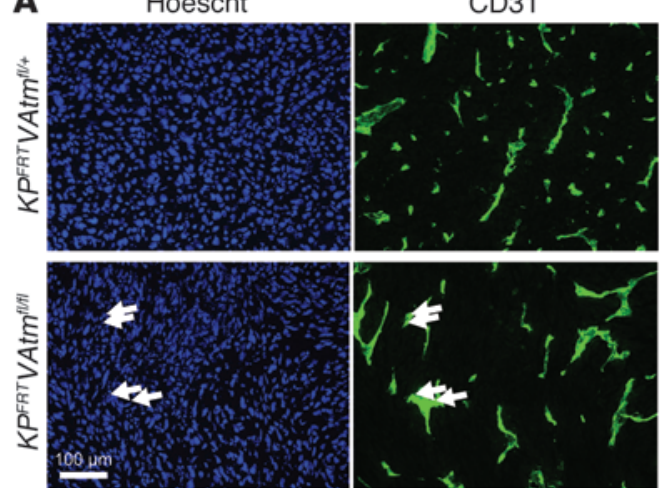

TUNEL
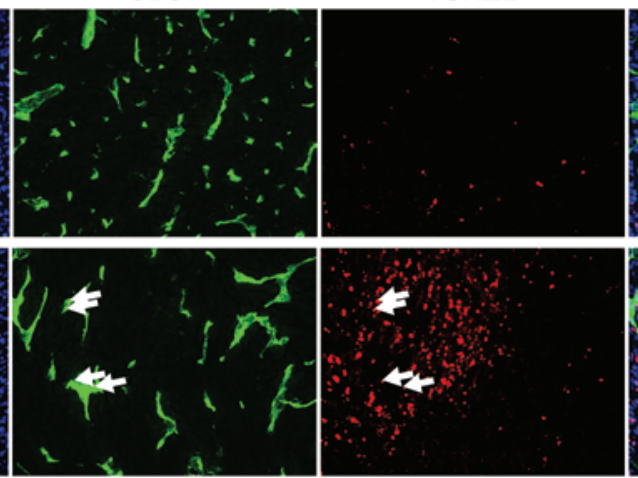

C

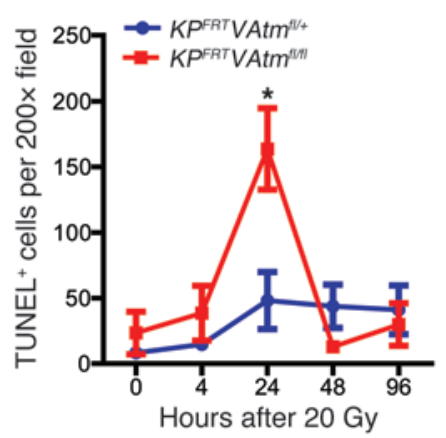

D

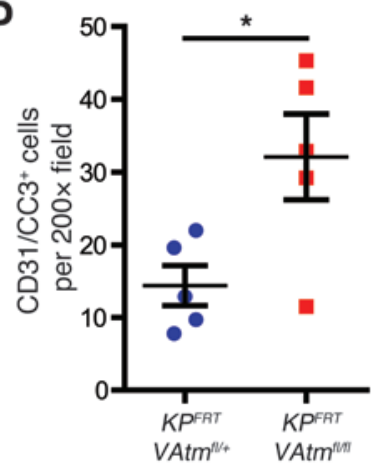

Merge

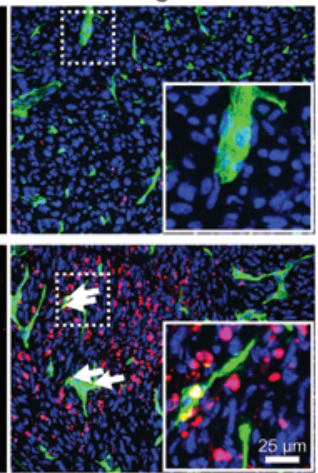

E

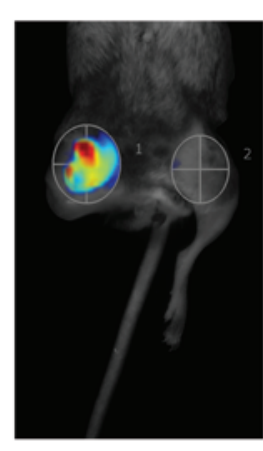

KPFRTVATM ${ }^{\text {II/+ }}$
B
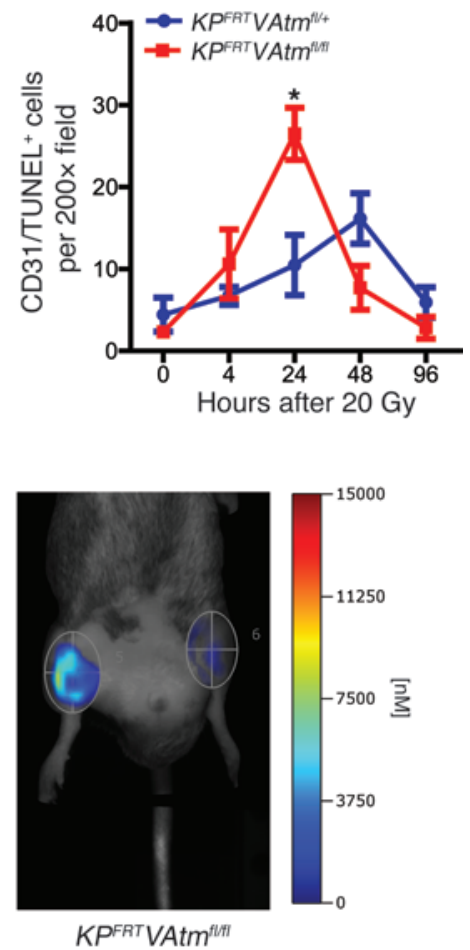

$\mathbf{F}$

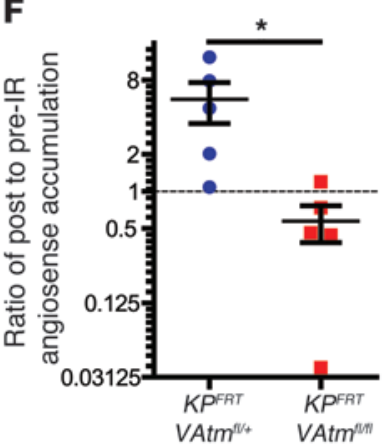

I

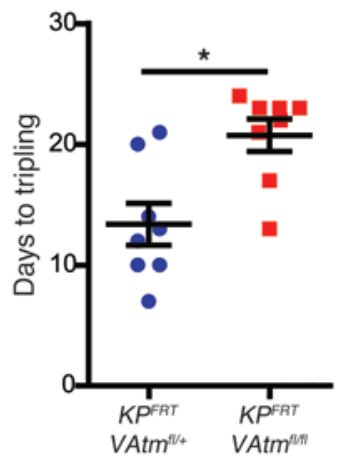

G

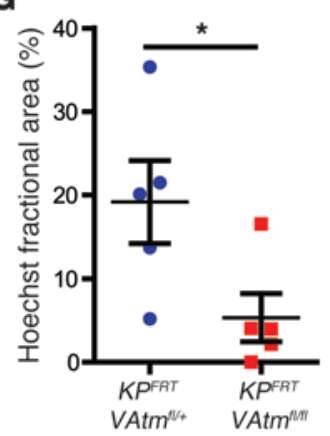

J

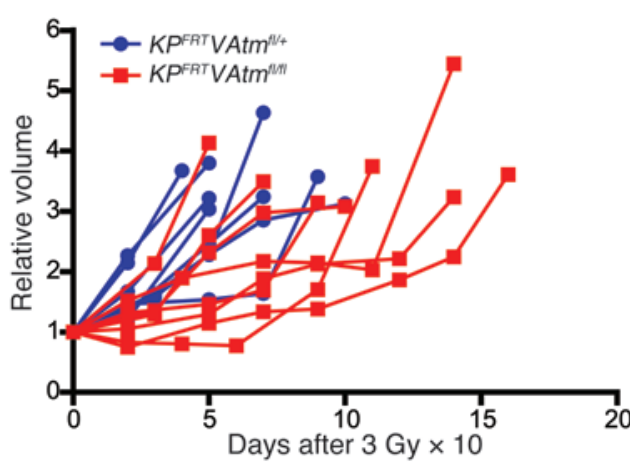

H
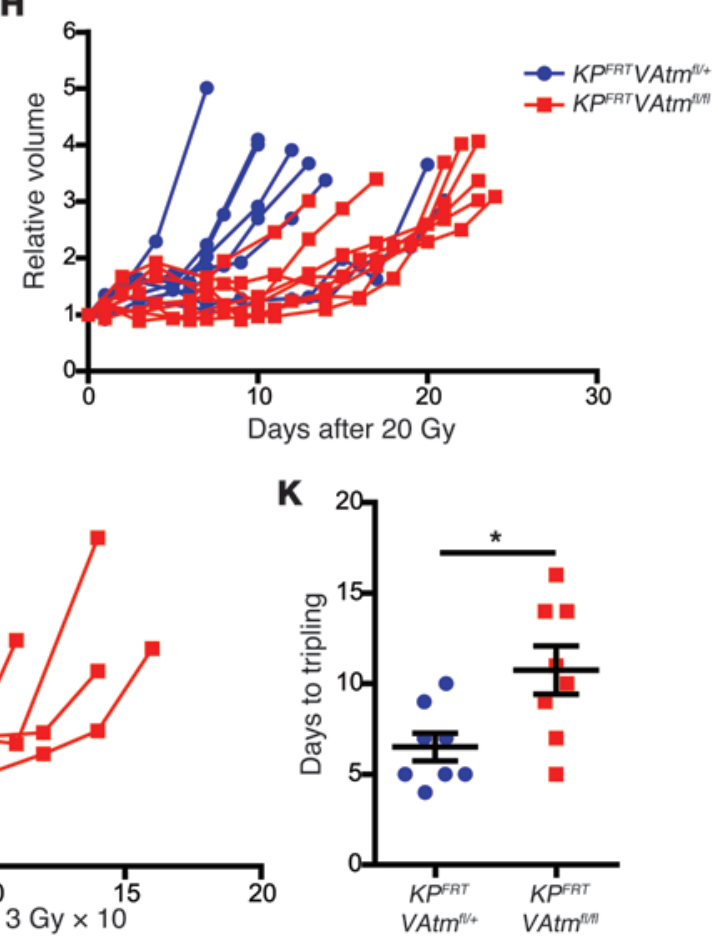

Figure 3. Loss of Atm sensitizes tumor endothelial cells to ionizing radiation and increases the radiation response of primary sarcomas. (A) Immunofluorescence for CD31 and TUNEL in sarcomas from $K P^{F R T} V A t m^{f /+}$ and $K P^{F R T} V A t m^{f / / l}$ mice 24 hours after irradiation with $20 \mathrm{~Gy}$. Examples of dead endothelial cells (white arrows) are shown at higher magnification in the insets. (B and C) Quantification of CD31+TUNEL ${ }^{+}$cells (B) and total TUNEL ${ }^{+}$cells (C) in sarcomas from KPFRTVAtm ${ }^{f /+}$ and $K P^{F R T} V A t m^{f / f l}$ mice at various time points after irradiation with $20 \mathrm{~Gy}\left(n=5\right.$ per group). (D) Quantification of CD31+CC3 ${ }^{+}$ cells in sarcomas from KPFRTVAtm ${ }^{f / /}$ and $K P^{F R T} V A t m^{f / / f l}$ mice 24 hours after irradiation with $20 \mathrm{~Gy}$ ( $n=5$ per group). (E) FMT of the blood pool imaging agent AngioSense, injected 24 hours after irradiation of sarcomas from $K P^{F R T} V A t m^{f /+}$ and $K P^{F R T} V A t m^{f / / f}$ mice with 20 Gy. (F) Quantification of the change in AngioSense accumulation after irradiation of sarcomas in $K P^{F R T} V A t m^{f /+}$ and $K P^{F R T} V A t m^{f / / 7}$ mice with $20 \mathrm{~Gy}$ ( $n=5$ per group). (G) Quantification of Hoechst 33342 perfusion in sarcomas from KPFRTVAtm ${ }^{f /+}$ and $K P^{F R T} V A t m^{f / f l}$ mice 24 hours after irradiation with $20 \mathrm{~Gy}$ ( $n=5$ per group). (H-K) Tumor growth curves and time to tripling for sarcomas in $K P^{F R T} V A t m^{f /+}$ and $K P^{F R T} V A t m^{f / f f}$ mice after irradiation with $20 \mathrm{~Gy}(\mathbf{H}$ and $\mathbf{I})$ or 10 daily fractions of $3 \mathrm{~Gy}(\mathbf{J}$ and $\mathbf{K})(n=8$ per group). All data are mean \pm SEM. ${ }^{*} P<0.05$. Scale bars: $100 \mu \mathrm{m}(\mathbf{A}) ; 25 \mu \mathrm{m}$ (A, insets). 
A

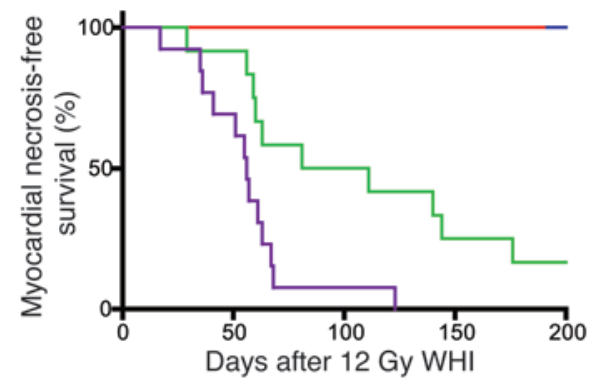

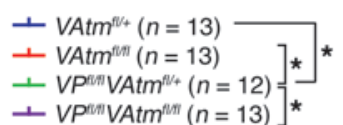

D

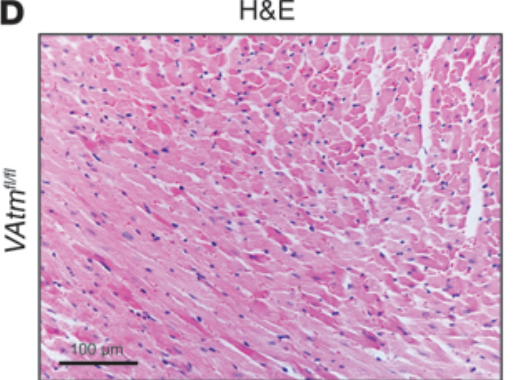

G

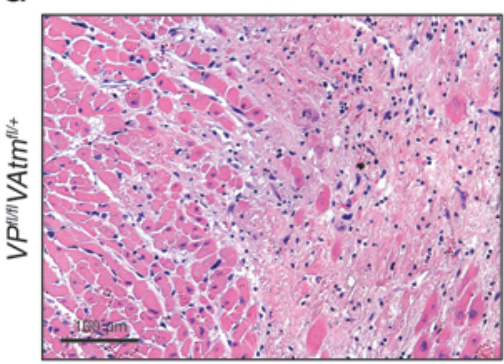

B

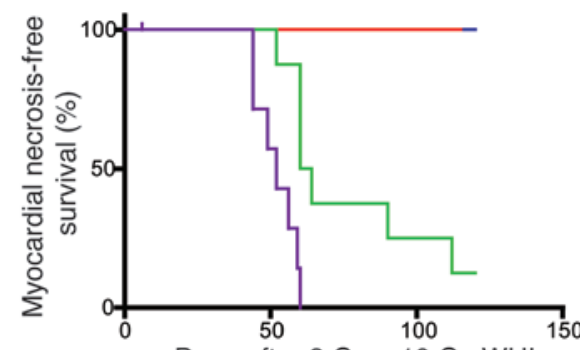

Days after 3 Gy $\times 10$ Gy WHI
C

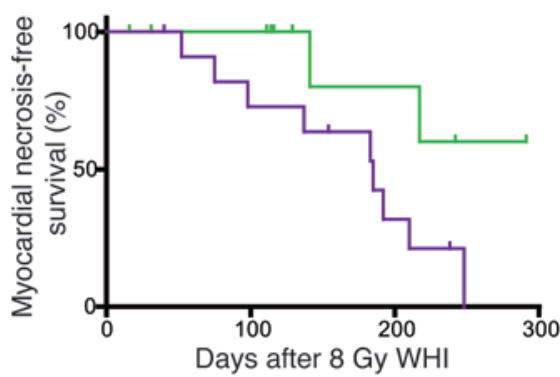

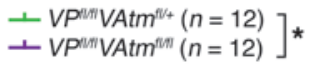

E

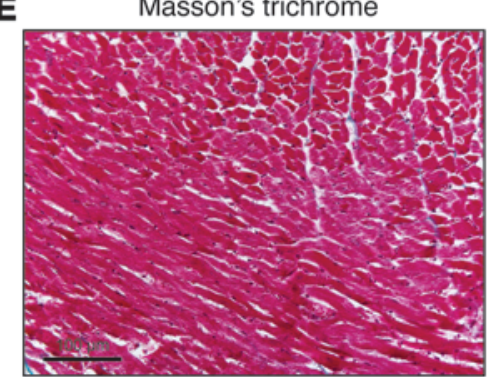

H

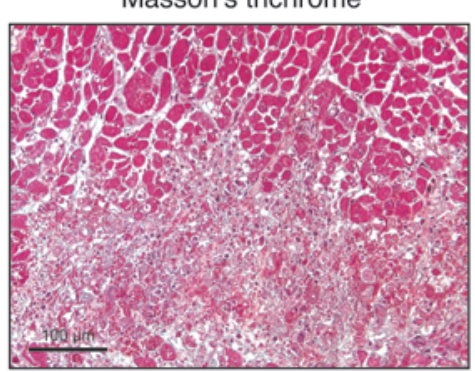

F Hoechst WGA TUNEL GS-IB ${ }_{4}$

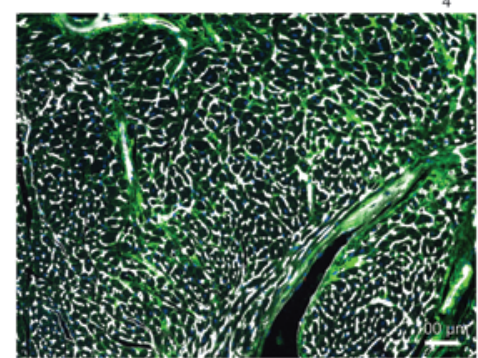

I Hoechst WGA TUNEL GS-IB ${ }_{4}$

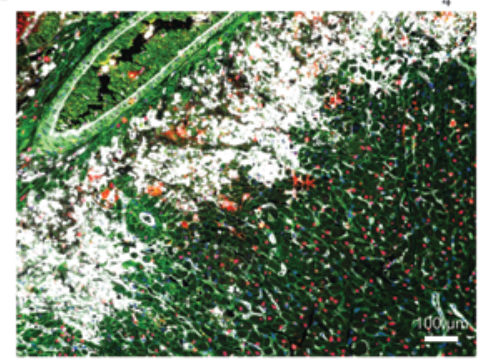

Figure 4. Deletion of Atm in p53 WT endothelial cells does not sensitize mice to radiation-induced myocardial necrosis. (A) Kaplan-Meier plots of myocardial necrosis-free survival for $V A t m^{f / /+}, V A t m^{f / f l}, V P^{f / / f} A t m^{f /+}$, and $V P^{f / / f} A t m^{f / f}$ mice after 12 Gy whole-heart irradiation. (B) Kaplan-Meier plots of myocardial necrosis-free survival for $V A t m^{f /+}, V A t m^{f / f l}, V P^{f / f} A t m^{f / /+}$, and $V P^{f / f} A t m^{f / f}$ mice after whole-heart irradiation with 10 daily fractions of $3 \mathrm{~Gy}$. $1 \mathrm{VP}^{f / f} \mathrm{Atm} / \mathrm{flfl}$ mouse died prior to finishing irradiation and was censored. (C) Kaplan-Meier plots of myocardial necrosis-free survival for $\mathrm{VP} / / / f / A t m^{f / /+}$ and $V P^{f / / f} A t m^{f / f l}$ mice after 8 Gy whole-heart irradiation. Mice of both genotypes were censored due to development of thymic lymphomas prior to heart disease. (D-I) Representative sections of the myocardium of $(\mathbf{D}-\mathbf{F})$ a $V A t m^{f / / f l}$ mouse 469 days after whole-heart irradiation and of $(\mathbf{G}-\mathbf{I})$ a $V P^{f / / f} A t m^{f / /+}$ mouse 56 days after whole-heart irradiation, subjected to staining with H\&E (D and $\mathbf{G})$ or Masson trichrome (E and $\mathbf{H})$ or immunofluorescence for WGA, TUNEL, and $\mathrm{CS}-\mathrm{IB}_{4}(\mathbf{F}$ and $\mathrm{I}) .{ }^{*} P<0.05$. Scale bars: $100 \mu \mathrm{m}(\mathbf{D}-\mathbf{I})$.

ing a radiation dose and fractionation schedule used in the clinic. Similar to the results with a single $20 \mathrm{~Gy}$ treatment, $K P^{F R T} V A t m^{A / f l}$ tumors had a significantly longer growth delay after fractionated radiation (Figure 3, J and $\mathrm{K}$ ). These findings further indicated that Atm deletion sensitizes tumor endothelial cells to radiation in vivo and demonstrated that endothelial cell death can contribute to the growth delay of primary tumors after radiation therapy.

Deletion of Atm in endothelial cells does not sensitize mice to radiation-induced myocardial necrosis. Damage to the vasculature of the heart after irradiation precedes pathological changes to the myocardium and contributes to radiation-induced cardiac injury (32). In response to ionizing radiation, ATM activates the transcription factor p53 through several mechanisms to stabilize $p 53$ mRNA and protein (5), which leads to increased transcription of p53 target genes, including the cyclin-dependent kinase inhibitor $p 21$. p21 is required for the radiation-induced G1 cell cycle checkpoint and for a sustained G2 checkpoint in some cells (33). Because we previously demonstrated that $p 21$-null mice or mice with VE-Cadherin-Cre-mediated deletion of $p 53$ are sensitized to radiation-induced myocardial necrosis within 4 months of $12 \mathrm{~Gy}$ whole-heart irradiation (11), we hypothesized that VE-CadherinCre; $\mathrm{Atm}^{f / f l}$ (referred to herein as VAtm ${ }^{f / f}$ ) mice would also succumb to myocardial necrosis after whole-heart irradiation.

To confirm that VE-Cadherin-Cre deletes Atm in heart endothelial cells, we isolated endothelial cells from $V A t m^{f / f l}$ and littermate control VAtm ${ }^{A /+}$ mice by FACS and measured Atm mRNA levels by qRT-PCR. Cardiac endothelial cells from VAtm ${ }^{A / f l}$ mice had significantly less Atm mRNA than did cardiac endothelial 
A

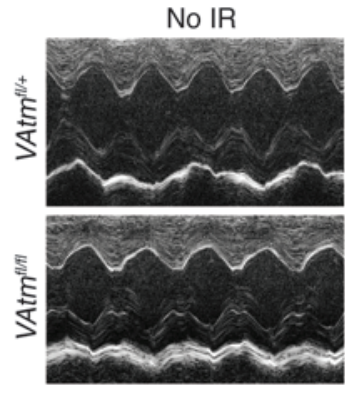

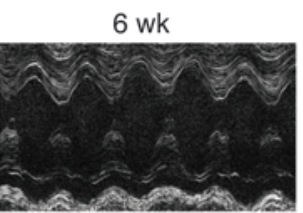

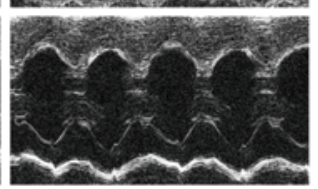

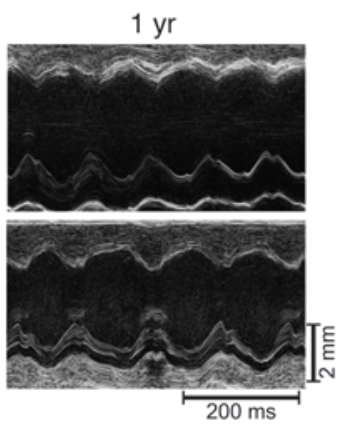

B

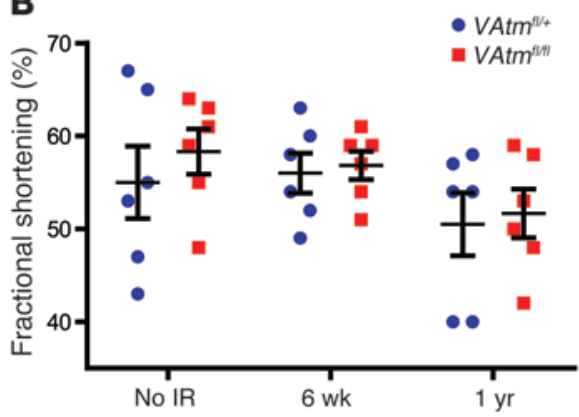

E

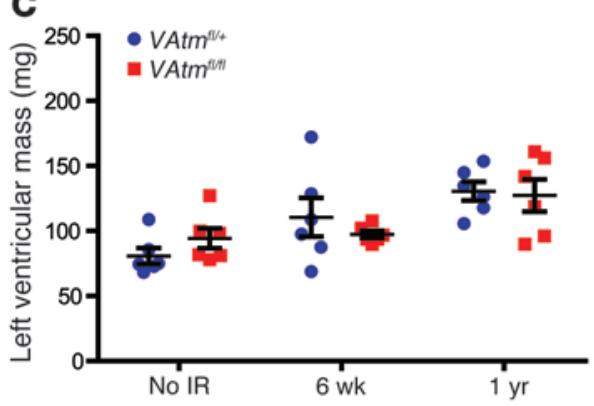

D

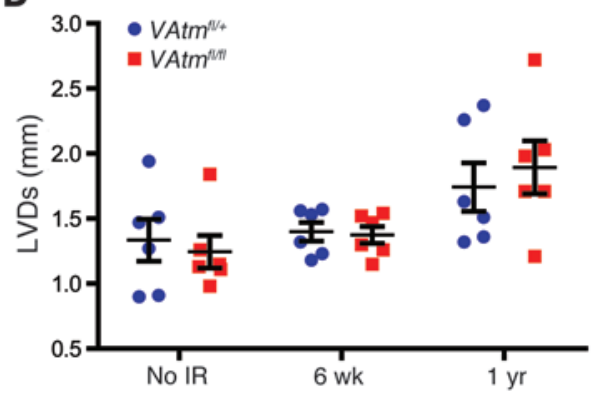

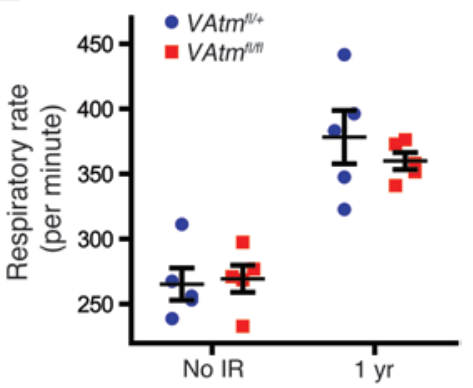

Figure 5. Characterization of cardiac and respiratory function in VAtm ${ }^{f /+}$ and VAtm ${ }^{f / f f}$ mice with and without whole-heart irradiation. (A) Representative echocardiography recordings from VAtm ${ }^{f /+}$ and VAtm ${ }^{f / f l}$ mice 6 weeks or 1 year after 12 Gy whole-heart irradiation and in unirradiated controls. (B-D) Changes in fractional shortening (B), left ventricular mass (C), and left ventricular end-systolic dimension (LVDs) (D) in VAtm ${ }^{f /+}$ and VAtm ${ }^{f / f l}$ mice 6 weeks and 1 year after 12 Gy whole-heart irradiation and in unirradiated controls ( $n=6$ mice per group). (E) Respiratory rate in VAtm ${ }^{f /+}$ and VAtm ${ }^{f / / f l}$ mice 1 year after 12 Gy whole-heart irradiation and in unirradiated controls ( $n=5$ per group). All data are mean \pm SEM.

cells from $V_{A t m}^{f /+}$ mice (Supplemental Figure 5A). We then irradiated $V A t m^{A / / A}$ and $V A t m^{f /+}$ mice with 12 Gy whole-heart irradiation (Supplemental Figure 5B). In contrast to tumor endothelial cells, deletion of Atm in heart endothelial cells did not increase the number of dead endothelial cells 24 hours after irradiation (Supplemental Figure 5, C and D).

Next, we irradiated $V_{A t m^{f /+}}$ and $V A t m^{f / f l}$ mice with a single dose of 12 Gy or 10 daily fractions of 3 Gy whole-heart irradiation and monitored them for development of myocardial necrosis (Figure 4, A and B). Remarkably, mice with deletion of Atm in endothelial cells did not die until 15 months after 12 Gy wholeheart irradiation (Figure 4A). Histopathological examination of the heart and lungs from both $V A t m^{f /+}$ and VAtm ${ }^{A / f}$ mice demonstrated focal regions of myocardial fibrosis and extensive pulmonary fibrosis, but no evidence of myocardial necrosis, after both single-dose and fractionated radiation exposure (Figure 4, D-F, and Supplemental Figure 6, A-H, M, and N). We did not observe any coronary atherosclerosis by histology after whole-heart irradiation of $V_{A t m}^{f /+}$ or $V A t m^{f / f l}$ mice.

To confirm that the difference in endothelial cell radiation sensitivity between tumors and hearts was not due to a difference in radiation dose, we also irradiated $V A t m^{f /+}$ and $V A t m^{f / A}$ mice with 20 Gy whole-heart irradiation. There was no difference in overall survival between the 2 genotypes (Supplemental Figure 7A). Histopathological examination demonstrated pulmonary hemorrhage and inflammation after 20 Gy whole-heart irradiation in both $V_{A t m^{f /+}}$ and VAtm ${ }^{f / f l}$ mice, but no evidence of myocardial necrosis (Supplemental Figure 7, B-E).
Because VE-Cadherin-Cre; $p 53^{f / A}$ mice are sensitized to radiation-induced myocardial necrosis, as a control, we next investigated whether loss of Atm in endothelial cells could further sensitize these mice to radiation-induced heart disease. We irradiated VE-Cadherin-Cre; $p 53^{A / f} ; A t m^{f / A l}$ (referred to herein as VP/f/l $\left.A t m^{A / f l}\right)$ mice and $V P^{f / f} A t m^{f /+}$ littermate controls with 10 daily fractions of 3 Gy or a single dose of 12 or 8 Gy whole-heart irradiation and monitored them for myocardial necrosis development. $V P^{f / f t}$ $A t m^{f / f l}$ mice exhibited significantly accelerated development of myocardial necrosis compared with $V P^{f / f t} \mathrm{Atm}^{f /+}$ mice after all radiation exposures (Figure $4, \mathrm{~A}-\mathrm{C}$ ). Histopathological examination of hearts from both $V P^{f / f} A t m^{f /+}$ and $V P^{f / f} A t m^{f / f}$ mice showed multifocal myocardial necrosis (Figure 4, G-I, and Supplemental Figure 6, I-L). These results suggested that Atm deletion sensitizes p53-null cardiac endothelial cells, but not cardiac endothelial cells with intact $p 53$, to radiation therapy.

To determine whether deletion of Atm in endothelial cells affects cardiac function after whole-heart irradiation, we performed echocardiography of $\mathrm{VAtm}^{f /+}$ and $\mathrm{VAtm}^{f / f l}$ mice prior to irradiation and 6 weeks and 1 year after whole-heart irradiation with 12 Gy. In contrast to mice with $p 53$ deleted in endothelial cells, which developed systolic dysfunction within 2 months after whole-heart irradiation (11), VAtm ${ }^{f /+}$ and VAtm ${ }^{f / f}$ mice maintained normal cardiac function 6 weeks after irradiation (Figure 5, A-D, and Supplemental Table 1). At 1 year after whole-heart irradiation, both $V A t m^{f /+}$ and VAtm ${ }^{A / f}$ mice began to develop cardiac dysfunction, manifest by decreased fractional shortening, increased left ventricular mass, and increased left ventricular end-systolic 
A
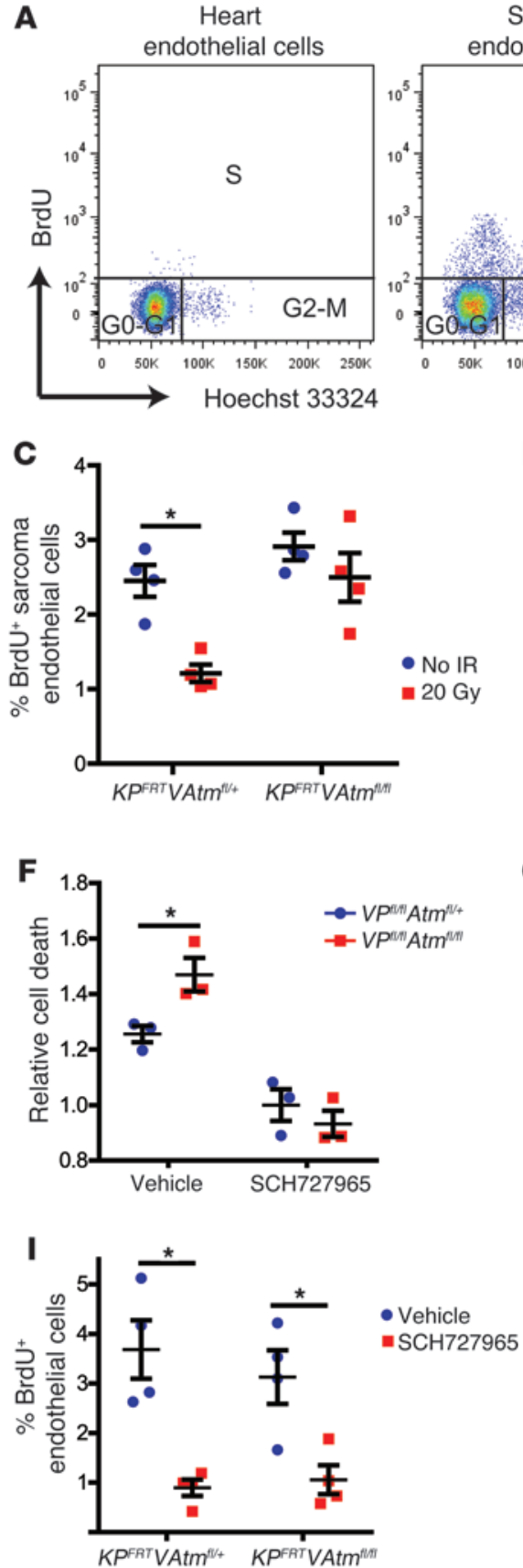

Sarcoma

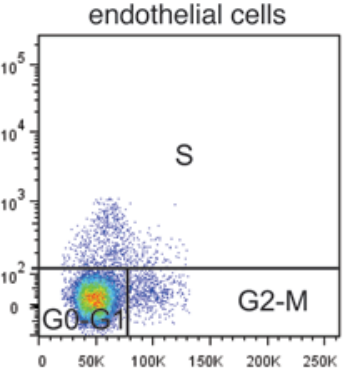

B

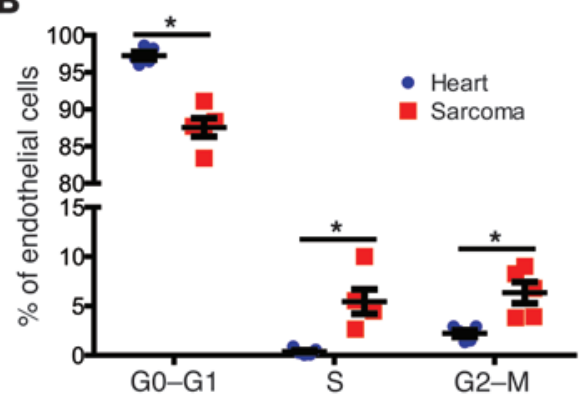

D

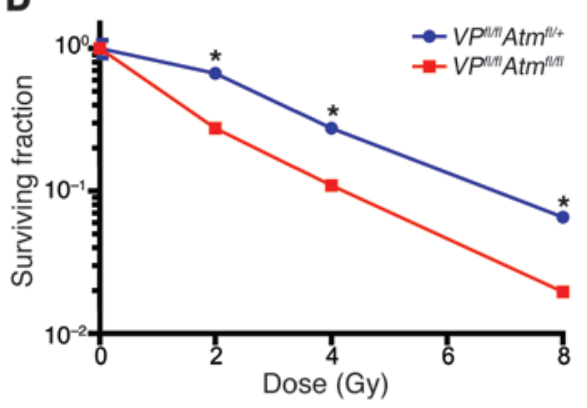

E

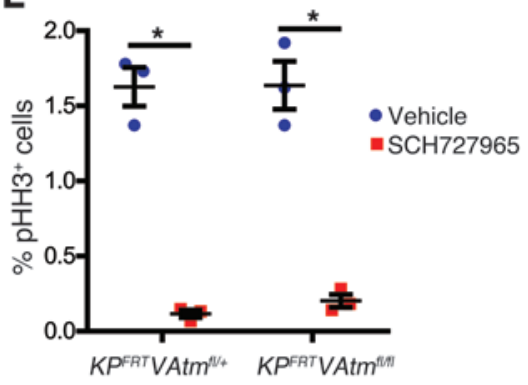

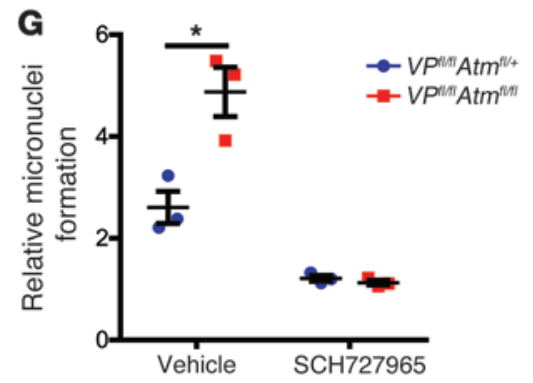

${ }_{\text {Ki67- }}^{H}{ }_{\text {Ki } 67^{+}}^{\text {* }}$

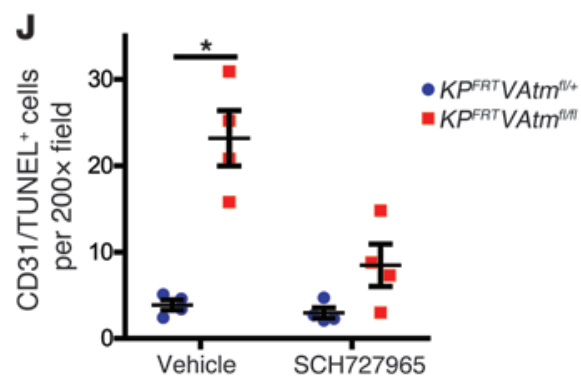

Figure 6. Atm deletion sensitizes proliferating endothelial cells to radiation. (A and B) Flow cytometry analysis (A) and quantification of cell cycle phase (B) in heart and sarcoma endothelial cells from $K P^{F R T}$ mice $(n=5)$. (C) Flow cytometry quantification of BrdU incorporation into tumor endothelial cells from $K P^{F R T}$ VAtm $^{f /+}$ and $K P^{F R T} V A t m^{f / f l}$ mice 1 hour after irradiation with 20 Gy or in unirradiated controls ( $n=4$ per group). (D) Clonogenic assay of primary cardiac endothelial cells from $V P^{f / / f} A t m^{f /+}$ and $V P^{f / f /} A t m^{f / f l}$ mice ( $n=3$ independent experiments). (E) Flow cytometry quantification of phosphorylated histone $\mathrm{H} 3$ (pHH3) for pri-

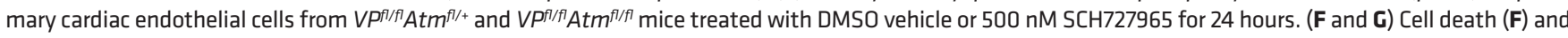

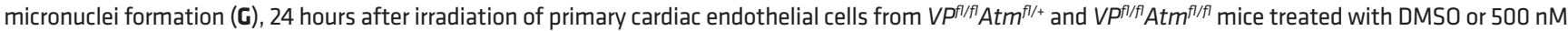
SCH727965 immediately before irradiation with $12 \mathrm{~Gy}(n=3$ independent experiments). Data are expressed relative to unirradiated cells of the same genotype and drug treatment. (H) Quantification of TUNEL staining in Ki67+ and Ki67- endothelial cells (CD31+) from tumors in $K P^{F R T} V A t m^{f / f l}$ mice 24 hours after irradiation with $20 \mathrm{~Gy}(n=5)$. (I) Flow cytometry quantification of BrdU incorporation into sarcoma endothelial cells from $K P^{F R T} V A t m^{f / /+}$ and $K P^{F R T} V A t m^{\text {fl/fI }}$ mice 24 hours after injection with vehicle or $40 \mathrm{mg} / \mathrm{kg}$ SCH727965 ( $n=4$ per group). (J) Quantification of CD31+TUNEL+ cells in sarcomas from $K P^{F R T} V A t m^{f / /+}$ and $K P^{F R T} V A t m^{f / f l}$ mice 24 hours after treatment with vehicle or SCH727965 immediately before irradiation with 20 Gy ( $n=4$ per group). All data are mean \pm SEM. ${ }^{*} P<0.05$.

dimension. Except for an increased aortic ejection time and a decreased heart rate in $V^{A t m^{A / A}}$ mice, there was not a significant difference in cardiac function between the 2 genotypes. Consistent with the pulmonary fibrosis observed histologically, both
$\mathrm{VAtm}^{A /+}$ and $\mathrm{VAtm}^{A / / A}$ mice had a markedly elevated respiratory rate (Figure 5E). Taken together with the histological studies, these results suggested that a combination of myocardial and pulmonary fibrosis contributed to the morbidity of $\mathrm{VAtm}^{A /+}$ and $\mathrm{VAtm}^{A / / \mathrm{A}}$ 
mice to a similar extent after 1 year. Therefore, deletion of Atm in endothelial cells does not affect the development of myocardial necrosis after whole-heart irradiation.

Loss of Atm sensitizes proliferating, but not quiescent, endothelial cells to radiation. Previous studies in mice demonstrated that deletion of Atm radiosensitizes proliferating normal tissues, such as the intestinal epithelium (12) and skin (34). We observed that although Atm deletion was sufficient to sensitize tumor endothelial cells to radiation, Atm deletion in cardiac endothelial cells with intact $p 53$ was not sufficient to sensitize mice to radiation-induced myocardial necrosis. Therefore, we hypothesized that the proliferative status of the endothelial cells might affect the impact of Atm loss on radiosensitivity.

Previous studies have shown that tumor endothelial cells proliferate much more rapidly than endothelial cells in normal tissues $(35,36)$. To determine whether endothelial cells proliferate more in primary sarcomas than in the heart, we compared the cell cycle distribution of endothelial cells in hearts and sarcomas in $K P^{F R T}$ $V_{A t m} / /++$ and $K P^{F R T} V A t m^{A / A}$ mice. In primary sarcomas, we observed significantly more endothelial cells in the S and G2-M phases and significantly fewer endothelial cells in G0-G1 compared with endothelial cells in hearts (Figure 6, A and B). In addition, BrdU pulse-chase analysis demonstrated that unlike heart endothelial cells, which did not undergo successive cell divisions within 48 hours, $\mathrm{BrdU}^{+}$tumor endothelial cells reenter the cell cycle within 24 hours (Supplemental Figure 8A). Deletion of Atm in endothelial cells did not affect the baseline proliferation of sarcoma or heart endothelial cells (Figure 6C and Supplemental Figure 8B). However, unlike sarcoma endothelial cells in $K P^{F R T} V A t m^{f /+}$ mice, those in $K P^{F R T} V A t^{f / / A}$ mice did not undergo cell cycle arrest after irradiation (Figure 6C). Taken together with our finding that deletion of Atm sensitized tumor - but not cardiac - endothelial cells to radiation, these results are consistent with a model in which proliferating endothelial cells are preferentially radiosensitized by Atm deletion.

To explore genes that may regulate the differential proliferation between sarcoma endothelial cells and heart endothelial cells, we performed qRT-PCR on FACS-isolated heart and sarcoma endothelial cells to determine mRNA levels of Atm, p53, p21, Ccnd1, and Ccnb1 (Supplemental Figure 8, C-G). Sarcoma endothelial cells had significantly less p21 mRNA than heart endothelial cells. Interestingly, both heart and sarcoma endothelial cells lacking Atm were able to significantly upregulate $p 21$ after radiation, but the level of $p 21 \mathrm{mRNA}$ in sarcoma endothelial cells after irradiation with 20 Gy was less than in unirradiated heart endothelial cells (Supplemental Figure 8, H and I).

To more directly assess the impact of proliferation on how Atm deletion affects endothelial cell death following radiation, we isolated primary cardiac endothelial cells from $V P^{f / f} A t m^{f /+}$ and $V P^{f / f l}$ $\mathrm{Atm}^{A / f}$ mice for in vitro studies (Supplemental Figure 9A). We also attempted to culture endothelial cells from VAtm ${ }^{f / f}$ mice (i.e., WT for p53), but primary cardiac endothelial cells without Atm failed to grow well in vitro. Therefore, our in vitro studies focused on $p 53-$ null endothelial cells. Cardiac endothelial cells from $V P^{f / f} A t m^{f / A l}$ mice were significantly more radiosensitive than those from $V P^{f / f l}$ $\mathrm{Atm}^{f /+}$ mice, as measured by clonogenic survival and flow cytometry for cell death (Figure 6D and Supplemental Figure 9B). Furthermore, deletion of Atm in cardiac endothelial cells significantly decreased in vitro tube formation and cell migration after irradiation (Supplemental Figure 9, C-F).

To investigate whether blocking cell cycle progression could protect these cardiac endothelial cells from the increased radiation sensitivity of Atm deletion, we treated the cells with the cyclindependent kinase 1 (CDK1), CDK2, CDK5, and CDK9 inhibitor SCH727965 (37). SCH727965 treatment for 24 hours significantly inhibited CDK activity, as measured by phosphorylation of retinoblastoma $(\mathrm{Rb})(38,39)$, and significantly decreased the progression of cardiac endothelial cells from both $V P^{f / f} A t m^{f /+}$ and $V P^{f / f} A t m^{f / f l}$ mice into mitosis (Figure 6E and Supplemental Figure 9, G and $\mathrm{H})$. Although vehicle-treated cardiac endothelial cells from $V P^{f / f t}$ $\mathrm{Atm}^{\mathrm{f} / \mathrm{fl}}$ mice underwent significantly more radiation-induced cell death 24 hours after radiation, there was not a significant change in cell death for cardiac endothelial cells from $V P^{f / f} \mathrm{Atm}^{f /+}$ and $V \mathrm{P}^{f / / f l}$ Atm $^{\text {f/fl/ }}$ mice treated with SCH727965 immediately prior to radiation exposure (Figure 6F). To determine whether the increased cell death in cardiac endothelial cells from $V P^{f / f} A t m^{f / f}$ mice was due to entry into mitosis with unrepaired DNA damage and subsequent mitotic catastrophe, we quantified micronuclei formation 24 hours after radiation of cardiac endothelial cells from $V P^{f / f} \mathrm{Atm}^{f /+}$ and $V P^{f / f} A_{t m}^{f / f l}$ mice with and without SCH727965 treatment. Vehicletreated cardiac endothelial cells from $V P^{f / f} A t m^{f / f}$ mice had significantly more micronuclei after irradiation with $12 \mathrm{~Gy}$, but there was not a significant change in micronuclei formation for cardiac endothelial cells from $V P^{A / f / A} A t m^{f /+}$ and $V P^{f / f} A t m^{f / A l}$ mice treated with SCH727965 immediately prior to radiation (Figure 6G).

To investigate whether deletion of Atm selectively radiosensitizes proliferating tumor endothelial cells to radiation in vivo, we stained sarcoma sections from $K P^{F R T} V A t m^{f / f l}$ mice 24 hours after radiation with CD31, TUNEL, and Ki67 to mark proliferating cells (40). There were significantly more $\mathrm{TUNEL}^{+}$endothelial cells in the $\mathrm{Ki} 67^{+}$population (Figure $6 \mathrm{H}$ ). Next, we treated tumor-bearing $K P^{F R T} V A t m^{f /+}$ and $K P^{F R T} V A t m^{t / f l}$ mice with vehicle or SCH727965 via intraperitoneal injection. SCH727965 treatment significantly decreased the number of $\mathrm{BrdU}^{+}$endothelial cells after 24 hours in both genotypes (Figure 6I). To determine whether blocking cell cycle progression could decrease the effect of Atm loss on tumor endothelial cell radiosensitivity in vivo, we quantified sarcoma endothelial cell death 24 hours after treatment of $K P^{F R T} V A t m^{A /+}$ and $K P^{F R T} V A t m^{A / f l}$ mice with vehicle or SCH727965 immediately prior to irradiation of the sarcomas with $20 \mathrm{~Gy}$. Consistent with the in vitro experiments, there was significantly more sarcoma endothelial cell death in $K P^{F R T} V A t m^{f / f l}$ mice after treatment with vehicle, but not SCH727965 (Figure 6J). Taken together, these results demonstrated that progression through the cell cycle is necessary for loss of Atm to radiosensitize endothelial cells.

\section{Discussion}

In this study, we used novel dual recombinase technology to compare the effect of Atm deletion on the response of the same cell type (endothelial cells) to radiation under different physiological states: proliferation and quiescence. We found that deletion of Atm in proliferating tumor endothelial cells enhanced the response of primary sarcomas to radiation, but deletion of Atm in quiescent endothelial cells of the heart did not sensitize mice to radiation-induced myocardial necrosis. Furthermore, blocking cell cycle progression 
reversed the effect of Atm deletion on endothelial cell radiosensitivity. These results indicated that endothelial cells must progress through the cell cycle to be radiosensitized by Atm deletion.

Previous studies have reported that loss of ATM does not affect the radiosensitivity of all cells and tissues equally. For example, most tissues in Atm-null mice appear histologically normal shortly after irradiation, but the rapidly proliferating cells in the intestine display severe radiation toxicity $(12,34,41)$. Interestingly, loss of $p 21$ further sensitizes Atm-null mice to radiation-induced gastrointestinal injury (42). Therefore, even in proliferative tissues, loss of cell cycle checkpoints may further increase the effect of Atm deletion on radiosensitization. Our present results suggested that the abrogation of cell cycle checkpoints allows quiescent cells to be sensitized to radiation as a consequence of Atm deletion. Recently, we reported that cardiac endothelial cells lacking $p 53$ or $p 21$ are sensitized to radiation (11). Furthermore, mice lacking $p 21$ or mice lacking $p 53$ specifically in endothelial cells were sensitized to radiation-induced myocardial necrosis (11). Here, we found that deletion of $p 53$ in endothelial cells not only permitted Atm deletion to sensitize endothelial cells to radiation in vitro, but also allowed Atm deletion to accelerate radiation-induced myocardial necrosis in vivo. Previous studies have reported that Atm deletion can sensitize other $p 53$-null cells and tissues to radiation $(43,44)$. By treating $p 53$-null endothelial cells in vitro or sarcoma endothelial cells in vivo with a cyclindependent kinase inhibitor at the time of irradiation, we were able to block the cells from undergoing mitotic catastrophe and limit the ability of Atm deletion to promote radiosensitivity. Taken together, these observations suggested that cells can be radiosensitized by targeting ATM, either because they are proliferating or because they have lost cell cycle checkpoints. These findings have important implications for combining ATM inhibitors with radiation therapy for treating human cancers, because tumor cells often proliferate rapidly and lack cell cycle checkpoints. Indeed, ATM knockdown or inhibition has been shown to preferentially sensitize $p 53$-null tumor cells to radiation or genotoxic chemotherapy $(9,45)$. Because proliferating normal cells are also sensitized by Atm deletion, the therapeutic window for targeting ATM will likely be larger for tumors located in or adjacent to quiescent normal tissues, such as extremity sarcomas and brain tumors.

We observed an increased aortic ejection time following whole-heart irradiation and an approximately $20 \%$ decrease in heart rate in mice with Atm deleted in endothelial cells. Therefore, even though deletion of Atm in endothelial cells did not affect survival or myocardial necrosis after whole-heart irradiation (Figure 4 and Supplemental Figure 7), it is possible that deletion of Atm in endothelial cells could have a mild effect on the sinoatrial node after irradiation. However, the relative effect of loss of Atm in endothelial cells on heart function after irradiation was much less than its effect on tumor response to radiation therapy. It should be noted that the mice used in this study were healthy and young at the time of whole-heart irradiation, and the effect of Atm deletion in endothelial cells on radiation-induced heart toxicity could be different in mice predisposed to develop heart disease. For example, vascular smooth muscle cells proliferate in atherosclerotic vessels (46), and targeting ATM may increase the sensitivity of these cells to radiation. As a result, targeting ATM could conceivably increase heart toxicity in patients with established vascular disease.
In this study, we focused on the effect of Atm deletion in endothelial cells on the development of radiation-induced myocardial necrosis. Although we previously showed that endothelial cell damage is sufficient to cause radiation-induced myocardial necrosis (11), it is likely that other cell types can also contribute to radiation-induced heart disease. In addition, the contribution of each cell type may depend on the dose and fractionation of radiation therapy. For example, radiation-induced fibrosis is a complex process that likely involves many different cell types (47). Moreover, the mechanisms of radiation injury likely vary across normal tissues. Therefore, the effect of targeting ATM during radiation therapy should be investigated in all clinically relevant tissues with different radiation doses and fractionation schemes.

In contrast to transplanted melanoma cell lines (18), deletion of Atm in the endothelial cells of primary soft tissue sarcomas did not affect vascular development or tumor growth. These discordant results may reflect differences between melanomas and sarcomas or differences in the tumor vasculature between tumors derived from cell lines and autochthonous tumors. Previous studies have demonstrated that the structure, oxygenation, perfusion, and response to therapy of tumor vasculature changes with transplantation $(19,48,49)$. Genetically engineered mouse models (GEMMs), such as the soft tissue sarcoma model used in the present study, develop within the native tumor microenvironment in immunocompetent mice (50) and may more faithfully recapitulate the tumor stroma and microenvironment of human cancer compared with xenograft models (51). Additionally, the response of these primary mouse cancer models to therapeutics has been shown to closely model the response of human cancers in clinical trials $(52,53)$. Taken together, these findings illustrate the importance of studying the tumor microenvironment using multiple complementary models, including GEMMs.

Most GEMMs use Cre recombinase to initiate cancer, making it challenging to simultaneously manipulate genes in tumor stromal cells. In this study, we used FlpO to initiate tumors, enabling VE-Cadherin-Cre to recombine Atm ${ }^{f}$ alleles in endothelial cells. Although FlpO and Cre have been used together previously to sequentially mutate genes in tumor cells (54), this is the first application of dual recombinase technology (FlpO and Cre) to simultaneously mutate genes in both tumor and stromal cells. Combining this dual recombinase technology with the many available floxed alleles and tissue-specific Cre drivers will be a powerful approach by which to investigate the contribution of stromal cells to cancer development, progression, and response to therapy.

It has been reported that endothelial cell apoptosis and microvascular collapse contribute to the response of transplanted tumors to radiation therapy (15). Endothelial cell apoptosis has been reported to be dependent on membrane damage, which triggers rapid ceramide-mediated apoptosis after high doses of radiation exposure $(55,56)$. In the present study, we did not observe substantial endothelial cell apoptosis at early time points after radiation exposure. Instead, endothelial cell death peaked at 24 hours after radiation in sarcomas from $K P^{F R T} V A t m^{A / f l}$ mice and 48 hours after radiation in sarcomas from $K P^{F R T} V A t m^{A /+}$ mice, which suggests that in primary soft tissue sarcomas, tumor endothelial cells die predominantly by a delayed mitotic cell death. Interestingly, the increased endothelial cell death in sarcomas 
from $K P^{F R T} V A t m^{f / f l}$ mice prolonged tumor growth delay after radiation therapy. Therefore, our results support a model whereby enhanced endothelial cell sensitivity to radiation increases tumor radiation response. We also observed that sarcomas in $K P^{F R T}$ $V_{A t m^{f / f l}}$ mice had decreased perfusion after radiation therapy. Although tumors in $K P^{F R T} V A t m^{A / A l}$ mice were also sensitized to irradiation with 10 fractions of $3 \mathrm{~Gy}$, it is possible that endothelial cell death may increase tumor hypoxia and thereby decrease the efficacy of subsequent fractions of radiation therapy. Furthermore, an increase in growth delay after radiation therapy does not necessarily translate into improved local control (57-59). To define the contribution of endothelial cells to local control after radiation therapy in this system, future experiments will be required with sarcomas in $K P^{F R T} V A t m^{f /+}$ and $K P^{F R T} V A t m^{f / f l}$ mice using higher doses of radiation and local control as the endpoint.

In summary, we found that deletion of Atm sensitized proliferating tumor endothelial cells to radiation and increased the response of primary soft tissue sarcomas to radiation therapy. In contrast, deletion of Atm in quiescent cardiac endothelial cells did not sensitize mice to radiation-induced myocardial necrosis. These results suggest that the state of cellular proliferation determines the effect of Atm deletion on radiosensitivity.

\section{Methods}

Mouse strains and tumor induction. All mouse strains used in this study were described previously, including LSL-Kras ${ }^{G 12 D}, p 53^{f}, F S F-K_{r a s}^{G 12 D}$, p53 ${ }^{F R T}, V E$-Cadherin-Cre, Atm ${ }^{f l}, m T m G$, and LSL-eYFP (20, 24-27, 54, $60,61) . L S L-K_{r a s}^{G 12 D}$ and FSF-Kras ${ }^{G 12 D}$ mice were provided by T. Jacks (Massachusetts Institute of Technology, Cambridge, Massachusetts, USA), $\mathrm{Atm}^{f}$ mice were provided by F. Alt (Harvard Medical School, Boston, Massachusetts, USA), and $p 53^{f t}$ mice were provided by A. Berns (The Netherlands Cancer Institute, Amsterdam, The Netherlands). VE-Cadherin-Cre, $m T M G$, and LSL-eYFP mice were obtained from the Jackson Laboratory. Primary sarcomas were generated in the right hind leg of $K P^{\text {loxP }}$ or $K P^{F R T}$ mice between 6 and 10 weeks of age, as described previously $(20,21)$. Whole-heart irradiation experiments were carried out with mice between 6 and 10 weeks of age. All mice were on a mixed genetic background. To minimize the effect of genetic background, age-matched littermate controls were used for every experiment so that potential genetic modifiers would be randomly distributed between the experimental and control groups.

Radiation treatment. Sarcoma and whole-heart irradiations were performed using the X-RAD 225Cx small animal image-guided irradiator (Precision X-Ray). The irradiation field was centered on the target via fluoroscopy with $40 \mathrm{kVp}, 2.5 \mathrm{~mA}$ X-rays using a $2 \mathrm{~mm} \mathrm{Al}$ filter. Mice were irradiated with parallel-opposed anterior and posterior fields with an average dose rate of $300 \mathrm{cGy} / \mathrm{min}$ prescribed to midplane with $225 \mathrm{kVp}, 13 \mathrm{~mA} \mathrm{X}$-rays using a $0.3 \mathrm{~mm} \mathrm{Cu}$ filter. Whole-heart irradiation was performed using a collimator to produce a $15-\mathrm{mm}$ circular radiation field at treatment isocenter. Sarcomas were irradiated at approximately $250 \mathrm{~mm}^{3}$ by caliper measurement using a collimator with a $40 \mathrm{~mm} \times 40 \mathrm{~mm}$ radiation field at treatment isocenter. After irradiation, sarcomas were measured 3 times per week until they tripled in size. Endothelial cells were irradiated in vitro with an X-RAD 320 biological irradiator (Precision $\mathrm{X}$-Ray). Cells were placed $50 \mathrm{~cm}$ from the radiation source and irradiated with a dose rate of $161 \mathrm{cGy} / \mathrm{min}$ using $320 \mathrm{kVp}, 10 \mathrm{~mA}$ X-rays and a $2 \mathrm{~mm} \mathrm{Al} \mathrm{filter.} \mathrm{Dose} \mathrm{rates} \mathrm{were} \mathrm{measured} \mathrm{with} \mathrm{an} \mathrm{ion} \mathrm{chamber}$ by the Radiation Safety Division at Duke University.

Histological analysis. Sarcoma histology was performed on frozen tissue sections. Tumor and heart frozen specimens were embedded directly in OCT compound (Sakura Finetek) by snap freezing in a dry ice/isopentane slurry and stored at $-80^{\circ} \mathrm{C}$ until sectioning. $10-\mu \mathrm{m} \mathrm{sec}-$ tions were fixed in $4 \%$ paraformaldehyde prior to immunofluorescence staining. For intracellular antigens, cells were permeabilized with $0.5 \%$ Tween 20 (Sigma-Aldrich). The primary antibodies used were rat antimouse CD31 (diluted 1:250; catalog no. 553370, BD Biosciences Pharmingen), rabbit anti-mouse Ser824-phosporylated KAP1 (diluted 1:250; catalog no. A300-767A, Bethyl Laboratories), polyclonal rabbit anti-mouse Ser1987-phosphorylated ATM (diluted 1:500; provided by M. Kastan, Duke University Medical Center, Durham, North Carolina, USA; ref. 62), rat anti-mouse VE-cadherin (diluted 1:250; catalog no. 555289, BD Biosciences - Pharmingen), rat anti-mouse CD34 (1:250, catalog no. 553731, BD Biosciences - Pharmingen), rat anti-mouse CD11b (diluted 1:250; catalog no. 14-0112-81, eBioscience), and rabbit anti-mouse CC3 (diluted 1:250; Cell Signaling, catalog no. 9661S). The secondary antibodies used were Alexa Fluor 488-conjugated donkey anti-rat IgG (diluted 1:500; catalog no. A21208, Invitrogen), Alexa Fluor 488-conjugated goat anti-rat IgG (diluted 1:500; catalog no. A11006, Invitrogen), Alexa Fluor 555-conjugated goat anti-rabbit IgG (diluted 1:250; catalog no. A21429, Invitrogen), and Alexa Fluor 647-conjugated goat anti-rat IgG (diluted 1:250; catalog no. A21247, Invitrogen). Nuclear staining was performed using Hoechst 33342 (10 $\mu \mathrm{M}$; Sigma-Aldrich).

Hypoxia was detected using the 2-nitroimidazole agent EF5. $10 \mathrm{mM}$ EF5 in PBS was administered through intraperitoneal injection at a volume of $26.5 \mu \mathrm{l} / \mathrm{g}$ body weight 3 hours prior to euthanasia and tissue collection. EF5 was detected by staining frozen sections with Cy3-conjugated anti-EF5 IgG. EF5 and Cy3-conjugated antiEF5 IgG were provided by C. Koch (University of Pennsylvania, Philadelphia, Pennsylvania, USA). Perfusion was detected by injecting $20 \mathrm{mg} / \mathrm{kg}$ Hoechst 33342 intravascularly 1 minute before tumor excision and snap freezing in OCT.

With the exception of CD31 and TUNEL staining, heart and lung histology was performed on paraffin-embedded tissue sections. Tissue specimens were fixed in $10 \%$ neutralized formalin overnight and preserved in $70 \%$ ethanol until paraffin embedding. 5- $\mu \mathrm{m}$ sections were deparaffinized with xylene and rehydrated with a graded series of ethanol and water washes prior to performing H\&E, Masson trichrome, or immunofluorescence staining. Antigens were retrieved using Antigen Unmasking Solution (Vector Laboratories) according to the manufacturer's instructions. Wheat germ agglutinin (WGA) and GS- $\mathrm{IB}_{4}$ staining was performed by incubating sections in $5 \mu \mathrm{g} / \mathrm{ml}$ Alexa Fluor 488-conjugated WGA (Invitrogen) and $10 \mu \mathrm{g} / \mathrm{ml}$ Alexa Fluor 647-conjugated GS-IB ${ }_{4}$ (Invitrogen) for 2 hours at room temperature.

TUNEL staining was performed on frozen sections with the In Situ Cell Death Detection Kit, TMR Red (Roche), according to the manufacturer's instructions.

Images were acquired with a Leica DFC340 FX fluorescence microscope (Leica Microsystems) using Leica Suite software (Leica Microsystems). Quantification of cells or fractional areas was performed by an observer blinded to genotype and treatment using Image (NIH). Each data point represents the average of 10 randomly selected $\times 200$ fields per sample. 
Flow sorting of tumor and heart endothelial cells. Tumors and hearts were dissected, washed in PBS, and homogenized. Tumors were digested in $5 \mathrm{mg} / \mathrm{ml}$ type IV collagenase (Gibco) and $1.3 \mathrm{mg} / \mathrm{ml}$ dispase (Gibco), and hearts were digested in $0.8 \mathrm{mg} / \mathrm{ml}$ type I collagenase (Worthington) for 1 hour at $37^{\circ} \mathrm{C}$. Digested tissues were filtered, and red blood cells were lysed with ACK lysing buffer (Lonza). Total number of cells was counted by Coulter counter (Beckman Coulter). $3 \times 10^{6}$ cells were stained with PE-conjugated anti-mouse CD31 (catalog no. 102407, Biolegend), PE-Cy5-conjugated anti-mouse CD45 (catalog no. 15-0451, eBioscience) and eFluor 660-conjugated antimouse CD34 (catalog no. 50-0341, eBioscience) antibodies. Dead cells were excluded by staining with 7-AAD (BD Biosciences - Pharmingen). Viable $\mathrm{CD}^{-} 5^{-}$and $\mathrm{CD} 31^{+} \mathrm{CD} 34^{+}$cells were sorted by FACSVantage (BD Biosciences - Pharmingen) and used for RNA isolation.

$q R T-P C R$. Total RNA was extracted from sorted heart and tumor endothelial cells with the RNAqueous-Micro Kit (Ambion), and reverse transcription was performed with the iScript cDNA Synthesis Kit (Bio-Rad). qRT-PCR was performed using Taqman universal PCR master mix (Applied Biosystems) and TaqMan Gene Expression Assay Mix (Applied Biosystems) for Atm (Mm01177459_m1), p53 (Mm01731290_g1), p21 (Mm00432448_m1), Ccnd1 (Mm00432359_ m1), Ccnb1 (Mm03053893_gH), or Hprt (Mm0446968_m1). Hprt was used as an internal control to correct for the concentration of cDNA in different samples. Each experiment was performed with 3 replicates per sample, and the results were averaged.

FMT. Baseline angiosense accumulation was measured prior to irradiation by injecting $150 \mu \mathrm{l}$ Angiosense 680EX (Perkin Elmer) via tail vein injection. After 24 hours, mice were imaged with an FMT2500LX (Perkin Elmer) molecular imaging device using the $680 \mathrm{~nm}$ laser channel. Accumulated angiosense within the sarcoma was quantified using TrueQuant Imaging Software (Perkin Elmer) and normalized to normal muscle in the contralateral limb. 24 hours after irradiation of the sarcoma with $20 \mathrm{~Gy}$, mice were reinjected with Angiosense 750EX (Perkin Elmer) and imaged after 24 hours using the 750 nm laser channel to quantify the change in angiosense accumulation.

Echocardiography and respiratory rate. Transthoracic echocardiography was performed on conscious mice for all groups with a Vevo 2100 high-resolution image system (VisualSonics) as described previously (11). Respiratory rate was counted visually using highspeed video recordings.

Cell cycle analysis of tumor and heart endothelial cells. Mice were injected intraperitoneally with $200 \mu \mathrm{l}$ of $5 \mathrm{mg} / \mathrm{ml}$ BrdU in PBS. After 4 hours or at the indicated time point, tumors and hearts were harvested and dissociated as described above. $3 \times 10^{6}$ cells were stained with PE-conjugated anti-mouse CD31 (catalog no. 102407, Biolegend), PE-Cy5-conjugated anti-mouse CD45 (catalog no. 15-0451, eBioscience), and eFluor 660-conjugated anti-mouse CD34 (catalog no. $50-0341$, eBioscience) antibodies. Cells were then fixed in $2 \%$ paraformaldehyde overnight at $4^{\circ} \mathrm{C}$. Fixed cells were permeabilized using the Cytofix/Cytoperm Fixation/Permeabilization Kit (BD Biosciences Pharmingen) according to the manufacturer's instructions. Cells were then incubated in $300 \mu \mathrm{g} / \mathrm{ml}$ DNase I (Sigma-Aldrich) for 1 hour at $37^{\circ} \mathrm{C}$ followed by staining with a FITC-conjugated anti-BrdU antibody (catalog no. 11-5071, eBioscience) according to the manufacturer's instructions.Cellswereresuspendedin $20 \mu \mathrm{g} / \mathrm{mlHoechst} 33342$ (SigmaAldrich), and data were collected by FACSCanto (BD Biosciences Pharmingen) and analyzed with Flowjo (Tree Star Inc.).
Primary endothelial cell culture. Cardiac endothelial cells were isolated as described previously (11) and cultured in DMEM with $25 \mathrm{mM}$ HEPES and $2 \mathrm{mM}$ L-glutamine (Gibco) supplemented with 20\% FBS, $100 \mu \mathrm{g} / \mathrm{ml}$ heparin, antibiotic-antimycotic, nonessential amino acids, sodium pyruvate, and $100 \mu \mathrm{g} / \mathrm{ml}$ endothelial cell growth stimulant (Biomedical Technologies) on cell culture dishes coated with $0.1 \%$ gelatin (Sigma-Aldrich). The purity of cardiac endothelial cells was assessed by flow cytometry with FITC-conjugated anti-mouse CD31 (catalog no. 11-0311, eBioscience) and PE-conjugated anti-mouse CD105 (catalog no. 12-1051, eBioscience) antibodies. Cardiac endothelial cells from passage 1-4 after purification were used for experiments.

Clonogenic survival, tubule formation, and cell migration. For clonogenic survival assays, cells were plated in triplicate and allowed to adhere overnight prior to irradiation. After development of colonies, cells were fixed with $70 \%$ ethanol, stained with Coomassie Brilliant Blue (Bio-Rad), rinsed with deionized water, and dried. Populations of $>50$ cells were counted as 1 colony, and surviving fractions were calculated relative to unirradiated controls.

For tube formation assays, primary cardiac endothelial cells were irradiated at approximately $80 \%$ confluency. Immediately after radiation, cells were harvested and plated at a density of 20,000 cells/ well in a 96-well plate coated with $100 \mu$ l Matrigel (BD Biosciences - Pharmingen) per well. After incubating for 4 hours, 3 images of each well were taken $(\times 100)$. The number of tubules per field was quantified and averaged for each well.

For cell migration assays, primary cardiac endothelial cells were plated on gelatin-coated 6-well plates and allowed to reach $80 \%$ confluency. 3 linear wounds were created per well using a $200 \mu$ l pipette tip, and the cells were irradiated. After 48 hours, cells were fixed with $70 \%$ ethanol and stained with Coomassie Brilliant Blue. The amount of closure was scored on a $0-100$ scale for 3 areas of each wound; values are averages for each well.

Quantification of cell death by flow cytometry. Primary cardiac endothelial cells were irradiated at approximately $60 \%$ confluency and harvested at the indicated time points after irradiation. Cells were washed with PBS and stained with $5 \mu \mathrm{g} / \mathrm{ml}$ propidium iodide (Sigma-Aldrich) and FITC-conjugated annexin V (catalog no. 556420, BD Biosciences - Pharmingen) according to the manufacturer's instructions. Data were collected by FACSCanto (BD Biosciences Pharmingen) and analyzed with FlowJo (Tree Star Inc.). Cell death was determined by quantifying the percentage of annexin $\mathrm{V}^{+}$cells relative to unirradiated controls.

SCH727965 treatment. Primary cardiac endothelial cells at $60 \%$ confluency were treated with DMSO or 500 nM SCH727965 (Selleckchem) for 24 hours. Cells were harvested and fixed with cold $70 \%$ ethanol, then stained with rabbit anti-mouse phosphorylated histone $\mathrm{H} 3$ (pHH3) primary antibody (diluted 1:500; catalog no. ab5176, Abcam) followed by Alexa Fluor 488-conjugated goat anti-rabbit IgG (diluted 1:1,000; catalog no. A11008, Invitrogen). DNA content was determined by staining cells with $50 \mu \mathrm{g} / \mathrm{ml}$ propidium iodide. Flow analysis for phosphorylated $\mathrm{Rb}$ was performed using rabbit anti-Rb phosphorylated at Ser807 and Ser811 primary antibody (diluted 1:400; catalog no. 8516S, Cell Signaling) and Alexa Fluor 488-conjugated goat antirabbit IgG secondary antibody to stain primary cardiac endothelial cells fixed and permeabilized using the Cytofix/Cytoperm Fixation/ Permeabilization Kit (BD Biosciences - Pharmingen) according to the manufacturer's instructions. 
For radiation experiments, cells were treated with DMSO or $500 \mathrm{nM} \mathrm{SCH727965} \mathrm{immediately} \mathrm{prior} \mathrm{to} \mathrm{radiation} \mathrm{and} \mathrm{harvested} \mathrm{at}$ 24 hours for analysis of cell death by flow cytometry or micronuclei quantification. Relative cell death or micronuclei formation was quantified by normalizing irradiated cells to unirradiated cells of the same genotype and drug treatment. Data were collected by FACSCanto (BD Biosciences - Pharmingen) and analyzed with FlowJo (Tree Star Inc.). Micronuclei were counted as described previously (11). For in vivo experiments, mice were treated with $40 \mathrm{mg} / \mathrm{kg}$ SCH727965 in $20 \%$ (2-hydroxypropyl)- $\beta$-cyclodextran via intraperitoneal injection immediately prior to irradiation.

Statistics. 2-tailed Student's $t$ test was performed to compare means of 2 groups, and 1-way ANOVA followed by Bonferroni post-hoc test was performed to compare means of 3 or more groups. 2-way ANOVA was performed to examine the interaction between genotype and treatment, followed by Bonferroni post-hoc tests for pairwise comparisons of individual treatments or genotypes. Non-normally distributed data were log-transformed before applying statistical tests. For survival studies, Kaplan-Meier analysis was performed followed by the log-rank test for statistical significance. A $P$ value less than 0.05 was considered significant. All calculations were performed using Prism 5 (GraphPad).

Study approval. All animal studies were performed in accordance with protocols approved by the Duke University IACUC.

\section{Acknowledgments}

We thank Frederick Alt for providing Atm ${ }^{\ell}$ mice, Tyler Jacks for providing $L S L-$ Kras $^{G 12 D}$ and FSF-Kras ${ }^{G 12 D}$ mice, Anton Berns for providing $p 53^{f}$ mice, Michael Kastan for providing antibody against pATM, Howard Rockman for assistance with cardiac phenotyping, and Loretta Woodlief and Lixia Luo for help with mouse care. This work was supported by the National Cancer Institute, NIH (grant nos. F30 CA177220 and R21 CA175839).

Address correspondence to: David G. Kirsch, Duke University Medical Center, Box 91006, Durham, North Carolina 27708, USA. Phone: 919.681.8605; E-mail: david.kirsch@duke.edu.
1. Delaney G, Jacob S, Featherstone C, Barton M. The role of radiotherapy in cancer treatment: estimating optimal utilization from a review of evidence-based clinical guidelines. Cancer. 2005;104(6):1129-1137.

2. Begg AC, Stewart FA, Vens C. Strategies to improve radiotherapy with targeted drugs. Nat Rev Cancer. 2011;11(4):239-253.

3. Moding EJ, Kastan MB, Kirsch DG. Strategies for optimizing the response of cancer and normal tissues to radiation. Nat Rev Drug Discov. 2013;12(7):526-542.

4. Shiloh Y. ATM and related protein kinases: safeguarding genome integrity. Nat Rev Cancer. 2003;3(3):155-168.

5. Kastan MB, Lim DS. The many substrates and functions of ATM. Nat Rev Mol Cell Biol. 2000;1(3):179-186.

6. Paterson MC, Smith PJ. Ataxia telangiectasia: an inherited human disorder involving hypersensitivity to ionizing radiation and related DNA-damaging chemicals. Annu Rev Genet. 1979;13:291-318.

7. Rainey MD, Charlton ME, Stanton RV, Kastan MB. Transient inhibition of ATM kinase is sufficient to enhance cellular sensitivity to ionizing radiation. Cancer Res. 2008;68(18):7466-7474.

8. Hickson I, et al. Identification and characterization of a novel and specific inhibitor of the ataxiatelangiectasia mutated kinase ATM. Cancer Res. 2004;64(24):9152-9159.

9. Biddlestone-Thorpe L, et al. ATM kinase inhibition preferentially sensitizes p53-mutant glioma to ionizing radiation. Clin Cancer Res. 2013;19(12):3189-3200.

10. Kirsch DG, et al. p53 controls radiation-induced gastrointestinal syndrome in mice independent of apoptosis. Science. 2010;327(5965):593-596.

11. Lee CL, et al. p53 functions in endothelial cells to prevent radiation-induced myocardial injury in mice. Sci Signal. 2012;5(234):ra52.

12. Barlow $\mathrm{C}$, et al. Atm-deficient mice: a paradigm of ataxia telangiectasia. Cell. 1996;86(1):159-171.

13. Xu Y, Ashley T, Brainerd EE, Bronson RT, Meyn MS,
Baltimore D. Targeted disruption of ATM leads to growth retardation, chromosomal fragmentation during meiosis, immune defects, and thymic lymphoma. Genes Dev. 1996;10(19):2411-2422.

14. Hanahan D, Weinberg RA. Hallmarks of cancer: the next generation. Cell. 2011;144(5):646-674.

15. Garcia-Barros M, et al. Tumor response to radiotherapy regulated by endothelial cell apoptosis. Science. 2003;300(5622):1155-1159.

16. Suit HD, Willers H. Comment on "Tumor response to radiotherapy regulated by endothelial cell apoptosis" (I). Science. 2003;302(5652):1894.

17. Brown M, et al. Comment on "Tumor response to radiotherapy regulated by endothelial cell apoptosis" (II). Science. 2003;302(5652):1894.

18. Okuno Y, Nakamura-Ishizu A, Otsu K, Suda T, Kubota Y. Pathological neoangiogenesis depends on oxidative stress regulation by ATM. Nat Med. 2012;18(8):1208-1216.

19. Falk P. Differences in vascular pattern between the spontaneous and the transplanted $\mathrm{C} 3 \mathrm{H}$ mouse mammary carcinoma. Eur JCancer Clin Oncol. 1982;18(2):155-165.

20. Lee CL, et al. Generation of primary tumors with Flp recombinase in FRT-flanked p53 mice. Dis Model Mech. 2012;5(3):397-402.

21. Kirsch DG, et al. A spatially and temporally restricted mouse model of soft tissue sarcoma. Nat Med. 2007;13(8):992-997.

22. Paris F, et al. Endothelial apoptosis as the primary lesion initiating intestinal radiation damage in mice. Science. 2001;293(5528):293-297.

23. White D, et al. The ATM substrate KAP1 controls DNA repair in heterochromatin: regulation by HP1 proteins and serine 473/824 phosphorylation. Mol Cancer Res. 2012;10(3):401-414.

24. Zha S, et al. Ataxia telangiectasia-mutated protein and DNA-dependent protein kinase have complementary V(D)J recombination functions. Proc Natl Acad Sci U S A. 2011;108(5):2028-2033.

25. Alva JA, et al. VE-Cadherin-Cre-recombinase transgenic mouse: a tool for lineage analysis and gene deletion in endothelial cells. Dev Dyn.
2006;235(3):759-767

26. Muzumdar MD, Tasic B, Miyamichi K, Li L, Luo L. A global double-fluorescent Cre reporter mouse. Genesis. 2007;45(9):593-605.

27. Srinivas $S$, et al. Cre reporter strains produced by targeted insertion of EYFP and ECFP into the ROSA26 locus. BMC Dev Biol. 2001;1:4.

28. Laitinen L. Griffonia simplicifolia lectins bind specifically to endothelial cells and some epithelial cells in mouse tissues. Histochem J. 1987;19(4):225-234.

29. Maddox DE, Shibata S, Goldstein IJ. Stimulated macrophages express a new glycoprotein receptor reactive with Griffonia simplicifolia I-B4 isolectin. Proc Natl Acad Sci U S A. 1982;79(1):166-170.

30. Vakifahmetoglu H, Olsson M, Zhivotovsky B. Death through a tragedy: mitotic catastrophe. Cell Death Differ. 2008;15(7):1153-1162.

31. Vasquez KO, Casavant C, Peterson JD. Quantitative whole body biodistribution of fluorescentlabeled agents by non-invasive tomographic imaging. PLoS One. 2011;6(6):e20594.

32. Stewart FA, Hoving S, Russell NS. Vascular damage as an underlying mechanism of cardiac and cerebral toxicity in irradiated cancer patients. Radiat Res. 2010;174(6):865-869.

33. Bunz F, et al. Requirement for p53 and p 21 to sustain G2 arrest after DNA damage. Science. 1998;282(5393):1497-1501.

34. Westphal CH, Rowan S, Schmaltz C, Elson A, Fisher DE, Leder P. atm and p53 cooperate in apoptosis suppression of tumorigenesis, but not in resistance to acute radiation toxicity. Nat Genet. 1997;16(4):397-401.

35. Hobson B, Denekamp J. Endothelial proliferation in tumours and normal tissues: continuous labelling studies. Br J Cancer. 1984;49(4):405-413.

36. Denekamp J, Hobson B. Endothelial-cell proliferation in experimental tumours. Br J Cancer. 1982;46(5):711-720.

37. Parry D, et al. Dinaciclib (SCH 727965), a novel potent cyclin-dependent kinase inhibitor. $\mathrm{Mol}$ Cancer Ther. 2010;9(8):2344-2353. 
38. Lin BT, Gruenwald S, Morla AO, Lee WH, Wang JY. Retinoblastoma cancer suppressor gene product is a substrate of the cell cycle regulator cdc2 kinase. EMBO J.1991;10(4):857-864.

39. Akiyama T, Ohuchi T, Sumida S, Matsumoto K, Toyoshima K. Phosphorylation of the retinoblastoma protein by cdk2. Proc Natl Acad Sci U S A. 1992;89(17):7900-7904.

40. Scholzen T, Gerdes J. The Ki-67 protein: from the known the unknown. JCell Physiol. 2000;182(3):311-322.

41. Ch'ang HJ, et al. ATM regulates target switching to escalating doses of radiation in the intestines. Nat Med. 2005;11(5):484-490.

42. Wang YA, Elson A, Leder P. Loss of p21 increases sensitivity to ionizing radiation and delays the onset of lymphoma in atm-deficient mice. Proc Natl Acad Sci U S A. 1997;94(26):14590-14595.

43. Takao N, et al. Disruption of ATM in p53-null cells causes multiple functional abnormalities in cellular response to ionizing radiation. Oncogene. 1999;18(50):7002-7009.

44. Westphal $\mathrm{CH}$, et al. Loss of atm radiosensitizes multiple p53 null tissues. Cancer Res. 1998;58(24):5637-5639.

45. Jiang H, et al. The combined status of ATM and p53 link tumor development with therapeutic response. Genes Dev. 2009;23(16):1895-1909.

46. Dzau VJ, Braun-Dullaeus RC, Sedding DG. Vascular proliferation and atherosclerosis: new perspectives and therapeutic strategies. Nat Med. 2002;8(11):1249-1256.
47. Bentzen S. Preventing or reducing late side effects of radiation therapy: radiobiology meets molecular pathology. Nat Rev Cancer. 2006;6(9):702-713.

48. Fenton BM, Lord EM, Paoni SF. Intravascular $\mathrm{HBO}(2)$ saturations, perfusion hypoxia in spontaneous transplanted tumor models. Int J Cancer. 2001;93(5):693-698.

49. Field SB, Needham S, Burney IA, Maxwell RJ, Coggle JE, Griffiths JR. Differences in vascular response between primary and transplanted tumours. Br J Cancer. 1991;63(5):723-726.

50. Sharpless NE, Depinho RA. The mighty mouse: genetically engineered mouse models in cancer drug development. Nat Rev Drug Discov. 2006;5(9):741-754.

51. Olive KP, et al. Inhibition of Hedgehog signaling enhances delivery of chemotherapy in a mouse model of pancreatic cancer. Science. 2009;324(5933):1457-1461.

52. Singh M, et al. Assessing therapeutic responses in Kras mutant cancers using genetically engineered mouse models. Nat Biotechnol. 2010;28(6):585-593.

53. Chen Z, et al. A murine lung cancer co-clinical trial identifies genetic modifiers of therapeutic response. Nature. 2012;483(7391):613-617.

54. Young NP, Crowley D, Jacks T. Uncoupling cancer mutations reveals critical timing of p53 loss in sarcomagenesis. Cancer Res. 2011;71(11):4040-4047.

55. Lin X, Fuks Z, Kolesnick R. Ceramide mediates radiation-induced death of endothelium. Crit Care Med. 2000;28(4 suppl):N87-N93.

56. Fuks Z, Kolesnick R. Engaging the vascular component of the tumor response. Cancer Cell. 2005;8(2):89-91.

57. Budach W, Taghian A, Freeman J, Gioioso D, Suit HD. Impact of stromal sensitivity on radiation response of tumors. J Natl Cancer Inst. 1993;85(12):988-993.

58. Krause M, et al. EGFR-TK inhibition before radiotherapy reduces tumour volume but does not improve local control: differential response of cancer stem cells and nontumourigenic cells? Radiother Oncol. 2007;83(3):316-325.

59. Zips D, et al. Impact of adjuvant inhibition of vascular endothelial growth factor receptor tyrosine kinases on tumor growth delay and local tumor control after fractionated irradiation in human squamous cell carcinomas in nude mice. Int $J$ Radiat Oncol Biol Phys. 2005;61(3):908-914.

60. Jackson EL, et al. Analysis of lung tumor initiation and progression using conditional expression of oncogenic K-ras. Genes Dev. 2001;15(24):3243-3248.

61. Jonkers J, Meuwissen R, van der Gulden H, Peterse H, van der Valk M, Berns A. Synergistic tumor suppressor activity of BRCA2 and p53 in a conditional mouse model for breast cancer. Nat Genet. 2001;29(4):418-425.

62. Valentin-Vega YA, et al. Mitochondrial dysfunction in ataxia-telangiectasia. Blood. 2012;119(6):1490-1500. 